\title{
La distance psychologique comme outil actionnable par les managers
}

\author{
Mohamed Didi Alaoui* \\ Université Côte d'Azur, IAE Nice \\ Groupe de Recherche en Management (GRM, EA 4711) \\ 5 rue du 22e B.C.A \\ 06300 Nice - France \\ mohamed.didi-alaoui@univ-cotedazur.fr
}

Véronique Cova

Aix Marseille Univ, IAE Aix, Aix-en-Provence

Centre d'Etudes et de Recherche en Gestion d'Aix-Marseille (CERGAM, EA 4425)

Chemin de la Quille Puyricard - CS 30063

13089 Aix-en-Provence - cedex 2 - France

veronique.cova@iae-aix.com

Article accepté pour publication dans Recherche et Applications en Marketing

Didi Alaoui, M., \& Cova, V. (2021). La distance psychologique comme outil actionnable par les managers. Recherche et Applications en Marketing.

https://doi.org/10.1177/0767370121992803

*Auteur correspondant 


\section{La distance psychologique comme outil actionnable par les managers}

\section{Résumé}

Cet article propose une synthèse des recherches ayant mobilisé le concept de distance psychologique autour des questions liées aux comportements prosociaux du consommateur, à la digitalisation de la consommation et aux pratiques marketing. Ce travail s'appuie sur une méta-synthèse de 584 articles issus de revues en psychologie et en marketing. Il permet une consolidation et une validation des connaissances du concept de distance psychologique dans ces différentes thématiques du marketing. Il montre également comment la distance psychologique peut être actionnée par les managers afin d'améliorer les réponses du consommateur. Les managers peuvent agir directement sur la distance en la réduisant ou l'augmentant pour profiter des bienfaits de la proximité ou de l'éloignement psychologique. Ils peuvent également ajuster les variables du marketing-mix en fonction de la distance psychologique expérimentée par le consommateur pour fluidifier le traitement de l'information et, in fine, améliorer les réponses de ce dernier.

\section{Mots-clés}

distance psychologique, théorie des niveaux de représentation, théorie du fit, comportements prosociaux, digitalisation, pratiques marketing 


\section{Introduction}

Quotidiennement, le consommateur expérimente différentes formes de proximité ou d'éloignement avec les stimuli de son environnement. C'est le cas lorsqu'il doit planifier un voyage qui aura lieu dans une semaine ou un an (distance temporelle), lorsqu'il perçoit dans l'espace marchand des produits qui sont proches ou éloignés physiquement de lui (distance spatiale), lorsqu'il décide d'acheter un produit pour lui-même ou une autre personne (distance sociale) ou lorsqu'il expérimente de l'incertitude ou de la certitude, comme gagner à un jeu de hasard (distance hypothétique). Ces différentes distances sont regroupées sous le concept de distance psychologique (Bar-Anan et al., 2007), qui est définie comme une expérience subjective associée au degré de proximité ou d'éloignement qu'un individu expérimente avec un stimulus par rapport au temps, à l'espace, au soi et à l'incertitude (Trope et Liberman, 2010). La distance psychologique peut être égocentrique lorsqu'elle concerne l'espace entre un individu et un stimulus (Yan, 2014) ou exocentrique lorsqu'il s'agit de l'espace entre deux stimuli (Liberman et Föster, 2009, Williams et Bargh, 2008). Ce concept a été développé par la théorie des niveaux de représentation (Construal Level Theory; CLT; Liberman et Trope, 1998 ; Trope et Liberman, 2010) selon laquelle le consommateur modifie son niveau de représentation, allant de l'abstrait au concret, lorsqu'il expérimente de la distance psychologique.

Omniprésente dans l'environnement et l'esprit du consommateur (Williams et al., 2014), la distance psychologique se présente comme une variable pertinente pour l'entreprise (Hamilton, 2014; Maglio, 2020 ; Malkoc et Zauberman, 2019), car les managers peuvent directement la réduire ou l'augmenter selon les réponses attendues du consommateur. Par exemple, l'offre Prime d'Amazon réduit la distance temporelle par un délai de livraison faible pour répondre favorablement à l'impatience du consommateur. Dans d'autres situations, les managers peuvent opter pour une augmentation de la distance afin d'accroître la désirabilité, la rareté et la perception de luxe du produit (Hansen et Wänke, 2011). Par exemple, Hermès propose une durée d'attente de trois ans avant d'obtenir un sac Kelly d'Hermès ${ }^{1}$.

La distance psychologique est également utile pour ajuster certains éléments du marketing-mix et améliorer leur efficacité. Par exemple, selon le degré de proximité (vs. éloignement) psychologique expérimenté par le consommateur, l'entreprise peut adapter ses arguments de vente en proposant un cadrage de communication négatif (vs. positif) (Balbo et Jeannot, 2015 ; White et al., 2011), comme le fait Betclic avec son slogan « NO BET NO GAME ». Ces ajustements créent chez le consommateur une perception d'adéquation (fit) qui fluidifie 
son traitement de l'information et améliore ses réponses (Roose et al., 2019 ; Spassova et Lee, 2013).

Dans la littérature, plusieurs articles de synthèse décrivent et expliquent le concept de distance psychologique dans le cadre de la CLT (Liberman, Trope et Wakslak, 2007 ; Liberman et Trope, 2008, 2014 ; Trope et al., 2007 ; Trope et Liberman, 2003, 2010 ; Wiesenfeld et al., 2017). Très récemment, Maglio (2020) a proposé une synthèse des antécédents et des conséquences de la distance psychologique perçue par le consommateur. Bien que ces différentes publications aient permis la dissémination des connaissances liées à ce concept, l'objectif ici est de consolider et présenter de façon structurée les travaux ayant mobilisé la distance psychologique dans trois thématiques : les comportements prosociaux, la digitalisation de la consommation et les pratiques marketing. Le choix de ces trois thématiques se justifie par leur importance dans le champ du marketing et par le très grand nombre de publications relatives à ces domaines. Aussi, structurer ces thématiques permet d'illustrer comment les managers peuvent utiliser la distance pour améliorer les réponses du consommateur.

Ce travail de consolidation se fonde sur une méta-synthèse de 584 articles publiés en psychologie et en marketing sur le concept de distance psychologique et dont la liste complète est disponible à cette adresse : https://urlr.me/c4KxY. La méta-synthèse vise à décomposer et examiner des résultats afin de faire apparaître les éléments fondamentaux d'une thématique (Beaucher et Jutras, 2007 ; Siau et Long, 2005). La sélection des articles a été réalisée à partir des bases de données transdisciplinaires suivantes: Ebsco, Emerald, Jstor, Sage, Scopus, Springer, Science Direct, Web of Science et Wiley. Nous avons utilisé les mots clés "psychological distance» et "construal level theory», ainsi que leur équivalent en français « distance psychologique » et « théorie des niveaux de représentation ». La période de publication des articles a été fixée entre 1998, date de l'article séminal de Liberman et Trope, et 2020. Notre recherche initiale a généré 7173 résultats. Nous avons écarté tous les articles de conférence, les doublons, les hors-sujet, les articles hors disciplines étudiées (marketing et psychologie) et ceux publiés dans une autre langue que l'anglais et le français. Nous n'avons pas intégré les articles portant uniquement sur les niveaux de représentation, et non sur la distance psychologique. À l'inverse, les articles qui portaient seulement sur la distance psychologique, sans se fonder sur les niveaux de représentation, ont été pris en compte.

Dans une première partie, nous présenterons la $C L T$ en montrant comment la distance psychologique et les niveaux de représentation ont été opérationnalisés en marketing. Puis, nous organiserons la littérature en trois thématiques: les comportements prosociaux du 
consommateur, la digitalisation de la consommation et les pratiques marketing. Nous conclurons en montrant les apports de notre analyse et les potentiels de futures recherches.

\section{Le cadre de la Construal Level Theory $(C L T)$}

\section{Les quatre dimensions de la distance psychologique}

Considérée comme un processus fondamental qui guide les cognitions des individus (Liberman et Trope, 2014), la distance psychologique a été développée dans le cadre de la CLT comme un concept regroupant quatre dimensions (Liberman et Trope, 2008). La distance temporelle, la première à avoir été étudiée, concerne les expériences futures (Liberman et al., 2002 ; Liberman et Trope, 1998 ; Pennington et Roese, 2003 ; Trope et Liberman, 2000) et les expériences passées (Caruso et al., 2013 ; Kyung et al., 2010, 2014). Par la suite, ont été examinées la distance spatiale (Fujita et al., 2006a ; Henderson et al., 2006), la distance sociale (Liviatan et al., 2008 ; Smith et Trope, 2006 ; Stephan et al., 2010) et la distance hypothétique (Todorov, et al., 2007 ; Wakslak et al., 2006). Ces travaux fondateurs de la CLT ont permis la retranscription de la distance psychologique en différentes situations managériales importantes pour la compréhension du comportement du consommateur.

Le tableau 1 présente la richesse des opérationnalisations qu'offre ce concept pour le marketing. Par exemple, la distance temporelle a été opérationnalisée selon la période de consommation du produit (dans un futur proche vs. lointain) (Jeannot et Jolibert, 2013). La distance spatiale correspond à la traduction géographique de situations de consommation spatialement proches ou éloignées dans l'esprit du consommateur (Merle et al., 2016). La distance sociale fait référence à des situations où le consommateur achète pour soi ou pour autrui (Baskin et al., 2014). Enfin, la distance hypothétique se définit comme la forte ou faible probabilité qu'un événement de consommation se réalise (Williams et al., 2014).

Bien que ces quatre dimensions traduisent chacune des formes de proximité ou d'éloignement spécifiques (Trope et Liberman, 2010), elles constituent un construit unitaire dans la mesure où elles sont unifiées sous une même appellation (Fiedler et al., 2012 ; Maglio et al., 2013a, 2013b), elles activent la même signification dans l'esprit de l'individu (c.-à-d. distance psychologique) (Bar-Anan et al., 2006) et elles influencent de la même manière les niveaux de représentation du consommateur (Bar-Anan et al., 2007), ses prédictions, évaluations, préférences et choix (Liberman et Trope, 2008 ; Trope et al., 2007).

Insérer le tableau 1 ici 


\section{Influence réciproque de la distance psychologique et du niveau de représentation du consommateur}

L'expérience avec différents objets peut se faire directement à travers nos sens, ou indirectement à travers une représentation mentale (Shapira et al., 2012), notamment lorsque les stimuli sont psychologiquement éloignés (Fujita et al., 2015 ; Liberman et Trope, 2014). La CLT avance que tout stimulus peut être construit sur l'un des deux niveaux de représentation suivants :

- Le haut niveau (high-level construal) : les représentations sont abstraites, globales, générales, cohérentes et décontextualisées.

- Le bas niveau (low-level construal) : les représentations sont concrètes, détaillées, précises, riches et contextualisées.

Lorsque le haut niveau est activé, le consommateur se focalise sur les éléments essentiels du stimulus. À l'inverse, lorsque le bas niveau est actif, le consommateur se concentre sur les éléments de détail (Liberman et Trope, 2008). Par exemple, l'objet « téléphone portable » sera représenté sur le haut niveau comme un « moyen de communication», alors que sur le bas niveau les représentations porteront sur des éléments de détail tels que la taille ou la couleur.

La CLT relie distance psychologique et niveaux de représentation : les stimuli psychologiquement éloignés ( $v s$. proches) sont représentés sur un haut ( $v s$. bas) niveau (Liberman et Trope, 2008 ; Trope et Liberman, 2010). Autrement dit, plus l'individu est éloigné (vs. proche) de l'objet, plus il tend à en construire une image globale, abstraite et décontextualisée ( $v s$. précise, détaillée et contextualisée).

Cette théorie montre également que la relation entre la distance psychologique et les niveaux de représentation est bidirectionnelle (Liberman et Trope, 2008). En effet, se représenter un stimulus sur un haut niveau ( $v$ s bas niveau) conduit l'individu à le percevoir comme étant psychologiquement éloigné (vs. proche) de l'ici, du maintenant, de soi et de la réalité (Henderson et al., 2011; Smith et al., 2008 ; Wakslak et Trope, 2009). Cette relation réciproque souligne d'une part l'importance qu'occupent les niveaux de représentation dans l'expérience de distance psychologique. D'autre part, elle montre que la distance peut être augmentée ou réduite selon que le consommateur adopte une vision large et globale, ou au contraire plus restreinte, localisée et précise du stimulus traité. Cependant, cette relation réciproque ne signifie pas que la distance psychologique et les niveaux de représentation sont 
interchangeables. De nombreuses recherches montrent qu'ils sont clairement distincts (Wang et al., 2019 ; Williams et al., 2014) et qu'ils peuvent avoir des effets opposés (Li et al., 2019).

En marketing, les niveaux de représentation ont été déclinés de différentes manières afin de déterminer quels types d'informations le consommateur sélectionne et utilise lorsqu'il expérimente de la distance psychologique dans son environnement d'achat. Comme le présente le tableau 2, plusieurs études démontrent que la proximité (vs. l'éloignement) psychologique conduit le consommateur à se focaliser sur les attributs concrets (vs. abstraits) d'un service (Pizzi et al., 2015), les aspects périphériques (vs. centraux) d'un produit (Trope et Liberman, 2000) et ses éléments de faisabilité (vs. désirabilité) (Liu, 2008). D'autres contributions avancent que la proximité ( $v s$. l'éloignement) conduit le consommateur à privilégier un mode visuel (vs. verbal) pour traiter un stimulus (Yan et al., 2016) et à se le représenter mentalement en couleur plutôt qu'en noir et blanc (Lee et al., 2017).

Ces différentes déclinaisons sont d'une grande utilité pour la compréhension du consommateur, car elles indiquent quelles informations il utilisera et traitera selon la distance psychologique qu'il expérimente. Ces déclinaisons peuvent également être utilisées pour réduire ou augmenter la distance psychologique expérimentée avec l'offre. Enfin, l'entreprise peut également s'en servir pour adapter ses variables du marketing-mix à la distance psychologique du consommateur.

Insérer le tableau 2 ici

\section{La distance psychologique pour éclairer le comportement du consommateur}

La distance psychologique pour comprendre l'écart entre attitudes et comportements prosociaux du consommateur

White et al. (2019) ont souligné l'écart existant entre les attitudes prosociales du consommateur et son comportement effectif. L'une des raisons qui expliquent cette différence est liée à la distance psychologique expérimentée avec des causes environnementales telles que le changement climatique (Brügger, 2020 ; Brügger et al., 2015 ; Spence et Pidgeon, 2010 ; Spence et al., 2012), la pollution (Zhang et al., 2014), la réduction du gaspillage (Barnes, 2019 ; Guillard, 2019 ), l'utilisation raisonnée des énergies (Griffioen et al., 2019 ; Tangari et Smith, 2012 ; Tangari et al., 2015) ou encore le recyclage (Schill et Schaw 2016 ; White et al., 2011).

Naturellement, lorsque le consommateur se sent psychologiquement éloigné d'une cause environnementale (ex : changement climatique), il tend à moins en visualiser le risque (ex : menace de certaines espèces) et les enjeux, ce qui se traduit le plus souvent par un manque 
d'engagement envers cette cause (Brügger et al., 2015 ; Scannell et Gifford, 2013 ; Spence et al., 2012). Ainsi, de nombreuses recherches suggèrent que la meilleure stratégie pour favoriser des comportements prosociaux est d'augmenter la proximité psychologique avec une cause environnementale (Ejelov et al., 2018 ; Jones et al., 2017 ; Leviston et al., 2014 ; Rickard et al., 2016 ; Scannel et Gifford, 2013). La proximité psychologique permettrait d'actionner plusieurs mécanismes, comme l'expérience d'émotions négatives (ex : peur, colère, tristesse ou culpabilité), la visualisation plus concrète des conséquences ainsi que le sentiment d'urgence lié à la situation écologique. Elle permet également au consommateur de se sentir davantage concerné et de vouloir agir en adoptant un comportement prosocial en cohérence avec ses attitudes (Shill et Schaw, 2016).

Cependant, le choix de la proximité ne s'avère pas systématiquement efficace (Manning et al., 2018 ; Schuldt et al., 2018 ; Spence et Pidgeon, 2010) et peut même détériorer les intentions comportementales du consommateur (Brügger, 2020 ; Brügger et al., 2016). En effet, en étant psychologiquement proche, l'individu se focalise spontanément sur la faisabilité (Liberman et Trope, 1998) des actions prosociales à mener, ce qui le conduit à surpondérer les coûts à consentir et sous-estimer les bénéfices qui sont rarement immédiats. La stratégie qui consiste à augmenter l'éloignement psychologique avec la cause peut s'avérer pertinente, car elle permet au consommateur d'élargir son horizon en se focalisant sur des valeurs globales (ex : la protection de la planète) et de vouloir agir positivement vis-à-vis de ses valeurs (Brügger et al., 2016 ; Corner et al., 2014). L'éloignement psychologique peut également favoriser la désirabilité liée aux bénéfices futurs et renforcer des réactions affectives positives comme l'espoir (Chu et Yang, 2019), qui a pour effet de stimuler la recherche de solutions et la mise en place d'actions collectives (Myers et al., 2012).

Ces résultats contrastés montrent que de nombreux modérateurs, comme l'identification résidentielle (résident local vs. citoyen du monde), le degré de pessimisme ou l'auto-efficacité perçue du consommateur, sont à considérer pour comprendre sous quelles conditions la distance psychologique peut réduire l'écart entre attitudes et comportements prosociaux. Par exemple, le fait que le consommateur se considère comme résident local ou comme citoyen du monde mitigera l'efficacité d'une stratégie visant à réduire ou augmenter la distance avec la cause environnementale (Brügger et al., 2015). En effet, si le consommateur a de fortes attaches avec l'endroit où il vit, alors un message qui mettra l'accent sur la proximité psychologique de la conséquence écologique (ex : «ça peut arriver près de chez vous ») conduira l'individu à se sentir davantage concerné et produira des effets positifs sur son action prosociale (Scannel et Gifford, 2013). A contrario, si l'individu se considère comme un citoyen du monde et semble 
être attaché à l'ensemble de la planète, alors un message qui souligne la proximité psychologique aura autant d'impact qu'un message qui accentue l'éloignement psychologique (ex : «L'Amazonie, une forêt tropicale en danger »).

Dans la même veine, le degré de pessimisme du consommateur peut expliquer l'efficacité de la proximité ou de l'éloignement psychologique. Certains consommateurs sont extrêmement pessimistes vis-à-vis de certaines causes environnementales et se montrent totalement désarmés face à ces phénomènes de grande ampleur (Leviston et al., 2014). Mettre l'accent sur la proximité psychologique aurait pour conséquence d'intensifier la menace et la peur liées à ce problème écologique et conduirait à des comportements de fuite pour réduire les affects négatifs (Brügger et al., 2016). Enfin, si le consommateur ne pense pas avoir les capacités suffisantes pour mettre en place des comportements prosociaux (auto-efficacité perçue), alors la proximité psychologique peut également le conduire à adopter des comportements de fuite ou de rejet pour réduire l'inconfort psychologique expérimenté (Poortinga et al., 2011). Ainsi, ces différentes recherches invitent les managers à étudier minutieusement leur cible à travers ces variables modératrices pour proposer une communication qui visera à réduire ou augmenter la distance psychologique avec la cause environnementale dans le but de réduire l'écart entre attitudes et comportements prosociaux.

\section{La distance psychologique comme variable clé pour décrypter les phénomènes de digitalisation de la consommation}

La digitalisation révolutionne les comportements d'achat (Forsythe et Shi, 2003 ; Grewal et al., 2020a ; Novak et al., 2003 ; Yoo et Donthu, 2001) en facilitant les échanges (Grewal et al., 2020b) et en offrant de nouvelles expériences au consommateur (Petit et al., 2019). L'enjeu du digital est de pouvoir offrir de la valeur au consommateur grâce aux nouvelles technologies, telles que les objets connectés (Hoffman et Novak, 2018 ; Novak et Hoffman, 2019), les robots (Belk, 2016 ; Goudey et Bonin, 2016), l'intelligence artificielle (Davenport et al., 2020) ou les réseaux sociaux (Borah et al., 2020 ; Kim et al., 2016), tout en préservant une proximité avec l'individu (Lenglet et Mencarelli, 2020).

Or, avec le digital, et notamment Internet, le consommateur expérimente de l'éloignement psychologique dans la mesure où il est très souvent spatialement et temporellement éloigné de l'offre proposée. De même, la privation de certains sens (toucher, odorat, goût) ainsi que l'expérience indirecte avec les produits (Melis et al. 2015 ; Yoon, 2002) renforcent cet éloignement. 
La distance expérimentée sur Internet peut avoir des effets négatifs sur le comportement d'achat, car l'éloignement psychologique tend à faire baisser la valeur perçue par un individu (Frederick et al., 2002 ; Malkoc et Zauberman, 2006). Ainsi, ce dernier préfère avoir immédiatement le produit plutôt que d'attendre et l'avoir plus tard (Laran, 2010a ; Nowlis et al., 2004). De plus, une forte distance psychologique réduit la prégnance (vividness) du produit, retarde la gratification immédiate du consommateur (Huygues et al., 2017 ; Shiv et Fedhorikin, 1999) et réduit l'importance attribuée aux éléments sensoriels (Fujita et al., 2006b ; Metcalfe et Mischel, 1999) et émotionnels de l'offre (Chang et Pham, 2013, 2018 ; Hong et Chang, 2015). Enfin, l'éloignement impacte négativement l'intensité des émotions positives (Van Boven et al., 2009, 2010 ; Williams et al., 2014), ce qui peut nuire à l'intention d'achat sur Internet des offres hédoniques caractérisées par une expérience affective et sensorielle positive (Huygues et al., 2017). Pour l'entreprise, l'enjeu est donc de réduire cet éloignement qui détériore l'expérience sensorielle et affective. Pour cela, elle peut recourir à de nombreuses technologies, comme l'InFORM shapeshifting display ${ }^{2}$ ou l'interface Shoogleit multi-gesture ${ }^{3}$, reproduisant des stimulations sensorielles capables de traduire une expérience directe avec le produit (Petit et al., 2019).

Par ailleurs, l'achat digital peut traduire une forte incertitude liée à l'alignement entre les attentes du consommateur et les réelles performances du produit. Ainsi, l'éloignement psychologique peut dégrader la confiance que le consommateur a envers le prestataire de l'offre, ce qui peut affaiblir ses intentions d'achat (Darke et al., 2016 ; Nguyen et al., 2020). Afin de gérer cette baisse de confiance, les managers peuvent par exemple proposer à leur consommateur, lorsqu'il expérimente de l'éloignement psychologique sur Internet, une garantie totale de la prestation plutôt qu'une garantie portant sur certains attributs de l'offre (Jin et He, 2013).

Toutefois, dans le contexte digital, l'éloignement psychologique peut avoir certains bienfaits, dans la mesure où il peut faciliter le processus de choix et réduire la procrastination des achats (Thomas et Tsai, 2012). En situation de proximité (contact physique avec l'offre), les représentations du consommateur sont de bas niveau : les informations à traiter vont dans le détail, ce qui complexifie les décisions d'achat. En situation d'éloignement (contact virtuel avec l'offre), l'individu est sur un haut niveau de représentation : les informations étant plus globales, elles occultent les difficultés, rendant ainsi la prise de décision plus aisée. De fait, dans le cas où l'entreprise souhaite faciliter la prise de décision du consommateur, il est judicieux de mettre en place des opérations qui augmenteront l'éloignement psychologique avec l'offre. 
Adéquation entre la distance psychologique et les outils du marketing-mix

L'efficacité des pratiques marketing est un enjeu majeur pour la croissance et la pérennité des entreprises (Homburg et al., 2020 ; Moorman et Day, 2016). Elle se traduit inévitablement par une adaptation et un excellent calibrage des quatre variables du marketingmix (McCarthy, 1964). La CLT a l'avantage d'offrir un cadre d'analyse robuste pour aiguiller l'entreprise dans le développement de son mix en fonction de la distance psychologique expérimentée par le consommateur. La littérature, abondante sur ce sujet, propose que les managers gagneraient à associer les modalités du mix qui activent un haut (vs. bas) niveau avec une situation d'éloignement ( $v s$. proximité) psychologique. Il ne s'agit plus de mettre en place une stratégie pour réduire ou augmenter la distance psychologique, mais plutôt de mettre en cohérence les variables du mix en fonction de cette dernière. Les avantages de cette mise en adéquation entre modalités du mix et distance psychologique sont justifiés par la théorie du fit (Higgins, 2000, 2005 ; Higgins et al., 2003) qui avance que l'adéquation ou la congruence entre deux modalités de deux variables permet de renforcer leur impact et d'améliorer in fine les réponses du consommateur (Freling et al., 2014).

Le produit. Pour se différencier dans des marchés de plus en plus concurrentiels, certaines marques accentuent la performance des éléments centraux de leur produit (ex : l'autonomie de l'Asus ROG Phone 3), tandis que d'autres dirigent leurs efforts sur des éléments périphériques comme des couleurs originales et distinctives (ex : le noir de jais d'Apple). Or, le consommateur manifeste souvent une instabilité dans ses préférences (Kopetz et al., 2012) liée à des facteurs contextuels comme la distance psychologique (Lu et al., 2016), ce qui complique la satisfaction de ses attentes. Ainsi, différentes recherches conseillent d'investir davantage d'efforts sur les caractéristiques de haut niveau du produit (ex : éléments centraux, caractéristiques liées à la désirabilité) lorsque la distance psychologique du consommateur est forte. À mesure que la distance se réduit et devient plus faible, il convient d'accentuer les éléments de bas niveau du produit (ex : éléments périphériques, caractéristiques liées à la faisabilité) (Baskin et al., 2014 ; Malkoc et al., 2005 ; Martin et al., 2009).

Un autre défi à relever pour les entreprises est de réussir le lancement des innovations pour minimiser les coûts marketing et de production (Jeannot et Jolibert, 2013). Cependant, le consommateur réagira différemment selon que l'innovation sera commercialisée dans longtemps (ex : voiture à hydrogène) ou que sa disponibilité est imminente (ex : la technologie 5G) (Alexander et al., 2008 ; Balbo et Jeannot, 2015 ; Ziamou et Veryzer, 2005). L’éloignement 
psychologique conduit l'individu à penser davantage aux bénéfices de l'innovation, alors que la proximité dirige l'attention du consommateur vers les coûts et les risques potentiels (Castaño et al., 2008). Ainsi, pour être en adéquation avec les niveaux de représentation que l'individu mobilise naturellement en situation d'éloignement ou de proximité psychologique, l'entreprise peut utiliser comme levier d'action marketing la simulation mentale (Jeannot et Jolibert, 2013 ; Lu et Jen, 2016; Zhao et al., 2007, 2011). Une simulation orientée vers le résultat et les bénéfices que l'innovation procure (c-à-d : activation du haut niveau) sera plus efficace en situation d'éloignement psychologique, tandis qu'une simulation du processus d'utilisation de l'innovation (c-à-d: activation du bas niveau) augmentera les intentions d'achat $d u$ consommateur en condition de proximité psychologique.

Le prix. Cet élément du mix représente une variable importante, car il permet à l'entreprise d'acquérir une part de la valeur créée (Deval et al., 2013). La littérature montre que le consommateur n'effectue pas les mêmes inférences liées aux prix selon la distance psychologique expérimentée. En situation de proximité, il tend à interpréter le prix comme un sacrifice monétaire, ce qui retranscrit la facilité ou la difficulté (faisabilité) d'accéder à l'offre (Bornemman et Homburg, 2011), alors qu'en situation d'éloignement le prix apparaît comme un gage de qualité et traduit la désirabilité de l'offre (Lee et Zhao, 2014 ; Yan et Segunpta, 2011). Ces résultats éclairent les pratiques d'entreprises en termes de positionnement prix. Lorsque le consommateur expérimente de la proximité psychologique, il semble judicieux pour l'entreprise de mettre l'accent sur l'accessibilité de l'offre ou sur un sacrifice monétaire préservé. À l'inverse, plus l'individu est éloigné de l'offre et plus un message portant sur l'inférence prix-qualité (ex : Mercedes-Benz: «le travail de qualité, c'est ma marque ») a de résonnance dans son esprit.

La promotion. La CLT suggère un ensemble d'opérationnalisations perceptuelles, conceptuelles et sensorielles ${ }^{4}$ pour guider l'entreprise dans l'adaptation de sa communication selon que le consommateur expérimente une distance psychologique forte ou faible. Pour les techniques perceptuelles, lorsque le consommateur est en situation d'éloignement (vs. proximité), l'entreprise peut améliorer ses réponses en utilisant davantage de texte plutôt que d'images (Amit et al., 2009, 2013, 2019 ; Choi et al., 2019 ; Torrez et al., 2019; Yan et al., 2016) ou en présentant la publicité en noir et blanc plutôt qu'en couleurs (Lee et al., 2017 ; Stillman et al., 2020 ; Wang et al., 2020). Elle peut également jouer sur le niveau d'horizon de la publicité en proposant un horizon bas (vs. haut) lorsque le consommateur est en condition d'éloignement (vs. proximité) (Roose et al., 2019). 
Pour les techniques dites conceptuelles, un cadrage positif (vs. négatif) de la communication améliore les réponses du consommateur en situation d'éloignement ( $v s$. proximité) psychologique (Balbo et Jeannot, 2015 ; 2017 ; Kulkarni et Yuan, 2015 ; 2017 ; White et al., 2011), tout comme une communication mettant davantage en exergue le « pourquoi » plutôt que le « comment» un produit sera consommé (Kim et al., 2018 ; Park et Park, 2016 ; Ryoo et al., 2017).

Enfin, l'entreprise peut recourir aux imageries sensorielles pour calibrer sa communication en fonction de la distance psychologique. Par exemple, la recherche d'Elder et al. (2017) montre que l'individu forme spontanément des imageries en lien avec des sens «distaux $»^{5}$ tels que l'ouïe, la vue ou l'odorat lorsqu'il imagine un objet éloigné psychologiquement. À l'inverse, lorsque l'objet est psychologiquement proche, ce sont des imageries liées au sens proximaux (ex : toucher, goût) qui sont davantage activées. En termes de communication, le message de l'entreprise sera d'autant plus persuasif s'il invite le consommateur en situation d'éloignement ( $v s$. proximité) à former une imagerie sensorielle qui se fonde sur des sens distaux (ex : « vous allez en prendre plein la vue ») plutôt que proximaux (ex : « vous allez en prendre plein les papilles »).

La place. En termes de distribution, l'un des enjeux pour les managers est d'offrir du choix au consommateur pour répondre à sa recherche de variété sans complexifier sa prise de décision (Goodman et Malkoc, 2012 ; Piris, 2013). Selon la distance psychologique expérimentée, le consommateur n'exprime pas toujours les mêmes préférences entre les assortiments selon leur taille. De nombreuses recherches montrent que l'individu apprécie fortement les assortiments larges (vs. restreints) en situation d'éloignement psychologique, car il accède à une capacité d'abstraction plus importante lui permettant de réduire la difficulté liée au choix entre les alternatives (Cho et al., 2013 ; Xu et al., 2013), d'améliorer sa prise de décision (Fukukura et al., 2013) et d'accroitre la désirabilité relative à un large choix (Goodman et Malkoc, 2012). Ainsi, dans des contextes où la distance psychologique est importante, comme l'achat sur Internet, l'entreprise peut se permettre d'élargir le nombre de ses alternatives sans affecter la difficulté du choix.

Cependant, cette recommandation n'est utile que lorsque le consommateur a conscience de la difficulté du choix liée à la taille de l'assortiment (Goodman et Malkoc, 2012). Dans le cas contraire, l'éloignement peut conduire l'individu à déprécier les assortiments de grande taille, car il percevra une forte similarité et redondance entre les alternatives proposées par l'entreprise (Henderson, 2013). 
La théorie $d u$ fit. Les différentes recherches qui encouragent les entreprises à adapter les variables du mix en fonction de la distance psychologique se fondent principalement sur la théorie du fit (Higgins, 2000, 2005 ; Higgins et al., 2003). En effet, mettre en adéquation les modalités de deux variables agissant en même temps augmente leur impact sur les réponses du consommateur (Teeny et al., 2020).

Selon la $C L T$ l'entreprise doit proposer une modalité du mix qui active un haut (vs. bas) niveau lorsque le consommateur perçoit de l'éloignement (vs. proximité) psychologique. Le tableau 3 mentionne les principales recherches fondées sur la théorie du fit pour prédire les effets positifs de l'adéquation entre les combinaisons haut niveau/éloignement et bas niveau/proximité.

Cet ajustement entre niveaux de représentation et distance psychologique offre au consommateur une expérience subjective de fluidité de traitement de l'information (Humphreys et al., 2020 ; Kim et al., 2009), qui a pour conséquence d'améliorer ses attitudes envers la publicité et la marque (Didi Alaoui, 2020; Spassova et Lee, 2013), son évaluation de la publicité (Roose et al., 2019; White et al., 2011), son intention d'achat et son consentement à payer un surprix pour l'offre proposée (Balbo et Jeannot, 2015).

Certaines recherches précisent que ces ajustements ne sont pas exclusivement expliqués par la fluidité de traitement, mais d'autres mécanismes, comme la perception de diagnosticité et d'utilité de l'attribut (Martin et al., 2009), de pertinence (Zhao et Xie, 2011), ou la vivacité des images mentales élaborées (Ding et Keh, 2017) médiatisent l'effet de l'adéquation entre l'une des modalités de la distance psychologique et une modalité du mix.

Insérer le tableau 3 ici

\section{Conclusion générale}

Cet article a pour principal objectif de structurer un grand nombre de recherches qui éclairent les managers sur la pertinence d'utiliser la distance psychologique pour mieux gérer les comportements prosociaux du consommateur, comprendre la digitalisation de la consommation et améliorer l'ajustement de leur marketing-mix. Bien que cette synthèse possède certaines limites, notamment liées à l'impossibilité d'intégrer toutes les contributions sur la distance psychologique en marketing, elle présente des contributions théoriques, des implications managériales et ouvre des perspectives de futures recherches. 


\section{Contributions théoriques}

Cette note de recherche contribue au continuum "découverte-justification » (Yadav, 2010) du cadre de la $C L T$, car elle permet de justifier la pertinence du concept de distance psychologique en montrant ce qui est valide et connu dans le champ du marketing. Dans la typologie des contributions conceptuelles proposées par MacInnis (2011), notre analyse poursuit un objectif d'explication en organisant une littérature abondante qui a développé un corpus riche de connaissances pour comprendre comment le consommateur se comporte lorsqu'il expérimente de la distance psychologique.

À la différence des synthèses précédentes (Trope et al., 2007 ; Maglio, 2020), cette recherche se focalise sur les trois domaines d'application qui nous ont paru porteurs et importants en marketing et qui ont suscité de nombreuses publications. Elle met notamment en lumière comment la distance psychologique permet d'expliquer l'écart entre attitude et comportements prosociaux. Elle insiste aussi sur la pertinence de la distance psychologique pour comprendre les phénomènes liés à la digitalisation de la consommation. Enfin, elle souligne l'importance de l'ajustement des variables du marketing-mix à la distance psychologique expérimentée par le consommateur. Cette consolidation des connaissances dans ces trois domaines regroupe ce que la littérature a découvert et validé empiriquement, offre des enseignements aux managers et ouvre des perspectives de futures recherches.

\section{Implications managériales}

Cette synthèse propose aux managers plusieurs éclairages pour mieux gérer la distance psychologique du consommateur en appliquant les stratégies les plus pertinentes au regard des connaissances établies par la littérature. Premièrement, l'entreprise peut se demander si elle souhaite uniquement agir sur la distance psychologique que le consommateur expérimente avec son offre. Si la réponse est positive, alors les managers peuvent actionner la distance psychologique en la réduisant (stratégie 1) lorsqu'elle occasionne des effets négatifs ou au contraire en l'augmentant (stratégie 2) lorsqu'elle améliore les réponses du consommateur. À titre d'exemple, si une ONG estime qu'une forte distance psychologique réduit l'implication envers une cause environnementale, alors des actions permettant de réduire la distance psychologique peuvent être mises en place. Cela peut être mené en accentuant la proximité temporelle (ex : «le réchauffement climatique, c'est maintenant que ça se passe »), spatiale (ex : « pensez à nos oliviers, n'imprimez ce mail que si nécessaire !»), sociale (ex : « respecter l'environnement, c'est offrir un avenir meilleur à son enfant ») ou hypothétique (ex : « $90 \%$ de 
chance que la fonte des glaces s'accélère cette année ») de la cause environnementale. Le tableau de l'annexe 1 présente des exemples d'actions que les entreprises peuvent mettre en place pour réduire ou augmenter la distance psychologique du consommateur.

Deuxièmement, les managers peuvent aussi faire le choix de ne pas agir directement sur la distance, mais d'ajuster les variables marketing en fonction de cette dernière. Dans cette phase, l'entreprise doit identifier la variable du mix à ajuster à la distance puis détecter la modalité du mix qui appartient au bas niveau et celle appartenant au haut niveau. Le tableau de l'annexe 2 propose trois méthodes, validées empiriquement par la littérature, pour guider l'entreprise dans la détection du niveau de représentation des modalités du mix. À la suite de cette phase, les managers doivent ajuster la modalité du mix appartenant au bas (haut) niveau à une situation de proximité (éloignement).

Par exemple, dans la publicité récente de l'Apple Watch Series $5^{6}$, 1'entreprise met en avant les différentes fonctionnalités de la montre (désirabilité). Cette mise en scène est pertinente pour les consommateurs qui envisagent d'effectuer leur achat sur Internet (c-à-d : en situation d'éloignement spatial), car c'est en adéquation avec l'activation du haut niveau de représentation. Cependant, cette publicité sera moins efficace pour les consommateurs qui souhaitent effectuer leur achat dans un Apple Store (c-à-d : en situation de proximité spatiale), car ces derniers activeront un bas niveau de représentation et seront davantage réceptifs à un message mettant en avant les éléments de faisabilité (facilité à utiliser la montre).

\section{Perspectives de recherche}

Cet article suggère également des pistes de recherche qui s'inscrivent dans les trois thématiques étudiées. La CLT a largement privilégié une opérationnalisation unidimensionnelle de la distance psychologique sans trop investiguer les effets de distances multiples (Huang et al., 2016 ; Kim et al., 2008). Or, dans de nombreuses situations, le consommateur expérimente plus d'une seule distance à la fois (Maglio et al., 2013a ; Yan, 2014).

Dans le cadre des comportements prosociaux, c'est notamment le cas lorsque le consommateur expérimente à la fois de la proximité spatiale et temporelle avec une cause environnementale (ex : les Maralpins avec la tempête Alex). Ainsi, des distances combinées réduisent-elles ou au contraire amplifient-elles l'écart entre attitude et comportement prosocial du consommateur? Si un effet de réduction ou d'amplification s'opère, ce dernier a-t-il la même magnitude lorsque deux, trois ou quatre dimensions de la distance psychologique sont combinées ? Quelles sont les influences possibles des modérateurs, tels que l'identification résidentielle (résident local vs. citoyen du monde), le degré de pessimisme ou l'auto-efficacité 
perçue du consommateur sur l'effet combiné de dimensions de la distance psychologique, sur l'écart entre attitude et comportement prosocial du consommateur?

Concernant la digitalisation de la consommation, l'individu peut expérimenter simultanément plusieurs distances appartenant à la même dimension, mais relatives à des éléments différents de la prise de décision du consommateur. Par exemple, lorsqu'il traite l'information d'une entreprise digitale éloignée spatialement de lui (ex : Facebook, Amazon), mais qui l'encourage à acheter chez un commerçant spatialement proche de lui (Google : « ensemble, continuons à soutenir les commerçants autour de chez nous »). C'est aussi le cas lorsqu'il commande un produit sur Internet qui sera livré 15 jours plus tard (éloignement temporel), alors que le paiement est débité instantanément (proximité temporelle). Comment la combinaison de ces deux distances appartenant à une même dimension (ex : spatiale) mais sur des modalités incongruentes (éloignement/proximité) influence-t-elle le comportement du consommateur?

Enfin, s'agissant de l'adéquation entre la distance psychologique et les outils du marketing-mix, l'entreprise peut vouloir ajuster une variable de son mix (ex : communication) pour le lancement d'un nouveau produit qui aura lieu dans un futur éloigné (c-à-d : éloignement temporel) et pour une nouvelle cible de consommateurs (c-à-d : éloignement social). Comment l'entreprise doit-elle gérer la combinaison entre la variable du mix et les différentes dimensions de la distance psychologique impliquées? Doit-elle opter pour une adéquation entre les modalités des trois variables ou alors une incongruence pourrait-elle, sous certaines conditions, produire des effets positifs ? 


\section{Remerciements}

Les auteurs remercient vivement le Rédacteur en chef, le Rédacteur en chef Associé ainsi que les trois lecteurs de RAM pour leurs conseils, remarques et suggestions précieuses.

\section{Notes}

\section{1. https://journalduluxe.fr/sac-kelly-hermes/}

2. L'InFORM shapeshifting display est une technologie permettant à l'individu de manipuler virtuellement et à distance des objets tangibles (voir par ex : Petit et al., 2019).

https://tangible.media.mit.edu/project/inform/

3. La technologie Shoogleit multi-gesture permet de reproduire des sensations de toucher d'un produit présenté virtuellement via un smartphone ou une tablette tactile (voir par exemple : Cano et al., 2017)

4. Nous distinguons les opérationnalisations conceptuelles qui portent sur la modification de concept (ex : positif $v s$. négatif), des opérationnalisations relatives aux perceptions (couleurs, images) et de celles liées aux sens (toucher, goût, odorat, vue, ouïe) (voir par exemple : Liberman et Förster, 2009).

5. Les sens distaux (ex : ouïe, vue, odorat) permettent de traiter les stimuli qui sont spatialement éloignés de l'individu alors que les sens proximaux ne peuvent être utilisés que si les stimuli sont spatialement proches de ce dernier (Elder et al., 2017).

\section{6. https://www.youtube.com/watch?v=siKJoFYwMMM}




\section{Références bibliographiques}

Aggarwal P et Zhao M (2015) Seeing the Big Picture: The effect of height on the level of construal. Journal of Marketing Research 52(1): 120-133.

Akpinar E, Verlegh PWJ et Smidts A (2018) Sharing product harm information: the effects of self-construal and self-relevance. International Journal of Research in Marketing 35(2): 319-335.

Alexander DL, Lynch JG et Wang Q (2008) As time goes by: do cold feet follow warm intentions for really new versus incrementally new products? Journal of Marketing Research 45(3): 307-319.

Amit E, Algom D et Trope Y (2009) Distance-dependent processing of pictures and words. Journal of Experimental Psychology: General 138(3): 400-415.

Amit E, Wakslak C et Trope Y (2013) The use of visual and verbal means of communication across psychological distance. Personality and Social Psychology Bulletin 39(1): 4356.

Amit E, Rim S, Halbeisen G, et al. (2019) Distance-dependent memory for pictures and words. Journal of Memory and Language 105: 119-130.

Balbo L et Jeannot F (2015) The fit between message framing and temporal distance: an efficient way to promote an innovative product. Recherche et Applications en Marketing (English Edition) 30(1): 28-49.

Balbo L, Jeannot F et Estarague J (2017) Promouvoir les comportements de santé pro-sociaux: l'association du cadrage du message et de la distance sociale. Décisions Marketing 85: $13-27$.

Bar-Anan Y, Liberman N et Trope Y (2006) The association between psychological distance and construal level: evidence from an implicit association test. Journal of Experimental Psychology: General 135(4): 609-622.

Bar-Anan Y, Liberman N, Trope Y, et al. (2007) Automatic processing of psychological distance: evidence from a Stroop task. Journal of Experimental Psychology: General 136(4): 610-622.

Barnes SJ (2019) Out of sight, out of mind: plastic waste exports, psychological distance and consumer plastic purchasing. Global Environmental Change-Human and Policy Dimensions 58: 1-9.

Baskin E, Wakslak CJ, Trope Y, et al. (2014) Why feasibility matters more to gift receivers than to givers: a construal-level approach to gift giving. Journal of Consumer Research 41(1): 169-182. 
Basoglu KA et Yoo JJ-E (2015) Soon or later? The effect of temporal distance on travel decisions. Journal of Travel \& Tourism Marketing 32: 62-75.

Beaucher V et Jutras F (2007) Étude comparative de la métasynthèse et de la méta-analyse qualitative. Recherches qualitatives 27(2): 58-77.

Belk R (2016) Comprendre le robot: commentaires sur Goudey et Bonnin (2016). Recherche et Applications en Marketing (French Edition) 31(4): 89-97.

Borah A, Banerjee S, Lin Y-T, et al. (2020) Improvised marketing interventions in social media. Journal of Marketing 84(2): 69-91.

Bornemann T et Homburg C (2011) Psychological distance and the dual role of price. Journal of Consumer Research 38(3): 490-504.

Borovoi L, Liberman N et Trope Y (2010) The effects of attractive but unattainable alternatives on the attractiveness of near and distant future menus. Judgment and Decision Making 5(2): 102-109.

Brügger A (2020) Understanding the psychological distance of climate change: the limitations of construal level theory and suggestions for alternative theoretical perspectives. Global Environmental Change 60: 1-7.

Brügger A, Dessai S, Devine-Wright P, et al. (2015) Psychological responses to the proximity of climate change. Nature climate change 5(12): 1031-1037.

Brügger A, Morton TA et Dessai S (2016) 'Proximising' climate change reconsidered: a construal level theory perspective. Journal of Environmental Psychology 46: 125-142.

Cano MB, Perry P, Ashman R, et al. (2017) The influence of image interactivity upon user engagement when using mobile touch screens. Computers in Human Behavior 77: 406-412.

Caruso EM, Van Boven L, Chin M, et al. (2013) The temporal Doppler effect: when the future feels closer than the past. Psychological science 24(4): 530-536.

Castaño R, Sujan M, Kacker M, et al. (2008) Managing consumer uncertainty in the adoption of new products: temporal distance and mental simulation. Journal of Marketing Research 45(3): 320-336.

Chang HH et Pham MT (2018) Affective boundaries of scope insensitivity. Journal of Consumer Research 45(2): 403-428.

Chang H, Zhang L et Xie G-X (2015) Message framing in green advertising: the effect of construal level and consumer environmental concern. International Journal of Advertising 34(1): 158-176.

Chang HH et Tuan Pham M (2013) Affect as a decision-making system of the present. Journal of Consumer Research 40(1): 42-63.

Chen F et Wyer RS (2015) The effects of affect, processing goals and temporal distance on information processing: qualifications on temporal construal theory. Journal of 
Consumer Psychology 25(2): 326-332.

Cho EK, Khan U et Dhar R (2013) Comparing apples to apples or apples to oranges: the role of mental representation in choice difficulty. Journal of Marketing Research 50(4): $505-516$.

Choi SY, Park HS et Oh JY (2012) Temporal distance and blood donation intention. Journal of Health Psychology 17(4): 590-599.

Choi WJ, Park J et Yoon H-J (2018) Your gift choice for your boss versus your subordinate would not be the same: the interplay of power and giver-receiver role on consumers' gift preferences. Journal of Business Research 91: 1-7.

Choi YK, Yoon S, Kim K, et al. (2019) Text versus pictures in advertising: effects of psychological distance and product type. International Journal of Advertising 38(4): 528-543.

Chu H et Yang JZ (2019) Emotion and the psychological distance of climate change. Science Communication 41(6): 761-789.

Corner A, Markowitz E et Pidgeon N (2014) Public engagement with climate change: the role of human values. Wiley Interdisciplinary Reviews Climate Change 5(3): 411-422.

Czeizler A et Garbarino E (2017) Give blood today or save lives tomorrow: matching decision and message construal level to maximize blood donation intentions. Health Marketing Quarterly 34(3): 175-186.

Darke PR, Brady MK, Benedicktus RL, et al. (2016) Feeling close from afar: the role of psychological distance in offsetting distrust in unfamiliar online retailers. Journal of Retailing 92(3): 287-299.

Davenport T, Guha A, Grewal D, et al. (2020) How artificial intelligence will change the future of marketing. Journal of the Academy of Marketing Science 48(1): 24-42.

Deval H, Mantel SP, Kardes FR, et al. (2013) How naive theories drive opposing inferences from the same information. Journal of Consumer Research 39(6): 1185-1201.

Didi Alaoui M (2020) L'effet d'adéquation entre la forme de présentation du prix et la distance temporelle sur les réponses du consommateur : une approche par la théorie des niveaux de représentation. Management Avenir 3: 41-62.

Ding Y et Keh HT (2017) Consumer reliance on intangible versus tangible attributes in service evaluation: the role of construal level. Journal of the Academy of Marketing Science 45(6): 848-865.

Ein-Gar D et Levontin L (2013) Giving from a distance: putting the charitable organization at the center of the donation appeal. Journal of Consumer Psychology 23(2): 197-211.

Ejelov E, Hansla A, Bergquist M, et al. (2018) Regulating emotional responses to climate change - a construal level perspective. Frontiers in Psychology 9: 1-8.

Elder RS, Schlosser AE, Poor M, et al. (2017) So close I can almost sense it: the interplay 
between sensory imagery and psychological distance. Journal of Consumer Research 44(4): 877-894.

Etkin J et Ratner RK (2013) Goal pursuit, now and later: temporal compatibility of different versus similar means. Journal of Consumer Research 39(5): 1085-1099.

Eyal T, Liberman N, Trope Y, et al. (2004) The pros and cons of temporally near and distant action. Journal of Personality and Social Psychology 86(6): 781-795.

Fiedler K, Jung J, Wänke M, et al. (2012) On the relations between distinct aspects of psychological distance: an ecological basis of construal-level theory. Journal of Experimental Social Psychology 48(5): 1014-1021.

Forsythe SM et Shi B (2003) Consumer patronage and risk perceptions in Internet shopping. Journal of Business research 56(11): 867-875.

Frederick S, Loewenstein G et O'donoghue T (2002) Time discounting and time preference: a critical review. Journal of economic literature 40(2): 351-401.

Freitas AL, Langsam KL, Clark S, et al. (2008) Seeing oneself in one's choices: construal level and self-pertinence of electoral and consumer decisions. Journal of Experimental Social Psychology 44(4): 1174-1179.

Freling TH, Vincent LH et Henard DH (2014) When not to accentuate the positive: reexamining valence effects in attribute framing. Organizational Behavior \& Human Decision Processes 124(2): 95-109.

Fujita K, Henderson MD, Eng J, et al. (2006a) Spatial distance and mental construal of social events. Psychological Science (0956-7976) 17(4): 278-282.

Fujita K, Trope Y, Liberman N, et al. (2006b) Construal levels and self-control. Journal of Personality and Social Psychology 90(3): 351-367.

Fujita K, Eyal T, Chaiken S, et al. (2008) Influencing attitudes toward near and distant objects. Journal of experimental social psychology 44(3): 562-572.

Fujita K, Trope Y et Liberman N (2015) On the psychology of near and far: a construal level theoretic approach. In: Keren G et Wu G (eds) The Wiley Blackwell Handbook of Judgment and Decision Making. John Wiley \& Sons, Ltd, pp. 404-430.

Fukukura J, Ferguson MJ et Fujita K (2013) Psychological distance can improve decision making under information overload via gist memory. Journal of Experimental Psychology: General 142(3): 658-665.

Gawronski B et Brannon SM (2020) Power and moral dilemma judgments: distinct effects of memory recall versus social roles. Journal of Experimental Social Psychology 86: 115.

Goodman JK et Malkoc SA (2012) Choosing here and now versus there and later: the moderating role of psychological distance on assortment size preferences. Journal of Consumer Research 39(4): 751-768. 
Goudey A et Bonnin G (2016) Un objet intelligent doit-il avoir l'air humain ? Etude de l'impact de l'anthropomorphisme d'un robot compagnon sur son acceptation. Recherche et Applications en Marketing (French Edition) 31(2): 3-22.

Grant SJ et Tybout AM (2008) The effect of temporal frame on information considered in new product evaluation: the role of uncertainty. Journal of Consumer Research 34(6): 897913.

Grewal D, Hulland J, Kopalle PK, et al. (2020a) The future of technology and marketing: a multidisciplinary perspective. Journal of the Academy of Marketing Science 48(1): 18.

Grewal D, Noble SM, Roggeveen AL, et al. (2020b) The future of in-store technology. Journal of the Academy of Marketing Science 48(1): 96-113.

Griffioen AM, Handgraaf MJJ et Antonides G (2019) Which construal level combinations generate the most effective interventions? A field experiment on energy conservation. PLoS One 14(1): 1-24.

Guillard V (2019) Le gaspillage perçu des objets : une analyse par les théories de la distance psychologique et des niveaux de représentation. Décisions Marketing, 93: 11-32.

Hamilton R (2014) Decisions at a distance: effects of psychological distance on consumer decision making. Journal of Consumer Research 41(2): iii-vi.

Hansen J et Wänke M (2011) The abstractness of luxury. Journal of Economic Psychology 32(5): 789-796.

Hernandez JM da C, Wright SA et Ferminiano Rodrigues F (2015) Attributes versus benefits: the role of construal levels and appeal type on the persuasiveness of marketing messages. Journal of Advertising 44(3): 243-253.

Henderson MD (2013) When seeing the forest reduces the need for trees: the role of construal level in attraction to choice. Journal of Experimental Social Psychology 49(4): 676683.

Henderson MD, Fujita K, Trope Y, et al. (2006) Transcending the 'here': the effect of spatial distance on social judgment. Journal of Personality and Social Psychology 91(5): 845856.

Henderson MD, Wakslak CJ, Fujita K, et al. (2011) Construal level theory and spatial distance implications for mental representation, judgment, and behavior. Social Psychology 42(3): 165-173.

Herzog SM, Hansen J et Wänke M (2007) Temporal distance and ease of retrieval. Journal of Experimental Social Psychology 43(3): 483-488.

Higgins ET (2000) Making a good decision: value from fit. American psychologist 55(11): 1217-1230.

Higgins ET (2005) Value from regulatory fit. Current directions in psychological science 14(4): 209-213. 
Higgins ET, Idson LC, Freitas AL, et al. (2003) Transfer of value from fit. Journal of Personality and Social Psychology 84(6): 1140-1153.

Hoffman DL et Novak TP (2018) Consumer and object experience in the internet of things: An assemblage theory approach. Journal of Consumer Research 44(6): 1178-1204.

Homburg C, Theel M et Hohenberg S (2020) Marketing excellence: nature, measurement, and investor valuations. Journal of Marketing 84(4): 1-22.

Hong J et Chang HH (2015) "I" follow my heart and "we" rely on reasons: the impact of selfconstrual on reliance on feelings versus reasons in decision making. Journal of Consumer Research 41(6): 1392-1411.

Huang N, Burtch G, Hong Y, et al. (2016) Effects of multiple psychological distances on construal and consumer evaluation: A field study of online reviews. Journal of Consumer Psychology 26(4): 474-482.

Huang Y, Jia Y et Wyer Jr. RS (2017) The effects of physical distance from a brand extension on the impact of brand-extension fit. Psychology \& Marketing 34(1): 59-69.

Humphreys A, Isaac MS et Wang RJ-H (2020) Construal matching in online search: applying text analysis to illuminate the consumer decision journey. Journal of Marketing Research. Epub ahead of print 2 september 2020. https://doi.org/10.1177\%2F0022243720940693

Huyghe E, Verstraeten J, Geuens M, et al. (2017) Clicks as a healthy alternative to bricks: how online grocery shopping reduces vice purchases. Journal of Marketing Research 54(1): $61-74$.

Jeannot $\mathrm{F}$ et Jolibert A (2013) Temporal distance, mental simulation and the adoption of complex technological innovations. Recherche et Applications en Marketing (English Edition) 28(1): 65-84.

Jha S, Deitz GD, Hart P, et al. (2019) Sales promotions for preorder products: the role of timeof-release. Psychology \& Marketing 36(9): 875-890.

Jia L et Smith ER (2013) Distance makes the metaphor grow stronger: a psychological distance model of metaphor use. Journal of Experimental Social Psychology 49(3): 492-497.

Jia Y, Huang Y, Wyer RS, et al. (2017) Physical proximity increases persuasive effectiveness through visual imagery. Journal of Consumer Psychology 27(4): 435-447.

Jin L et He Y (2013) Designing service guarantees with construal fit: effects of temporal distance on consumer responses to service guarantees. Journal of Service Research 16(2): 202-215.

Jin L, Hu B et He Y (2014) The recent versus the out-dated: an experimental examination of the time-variant effects of online consumer reviews. Journal of Retailing 90(4): 552566.

Jones C, Hine DW et Marks ADG (2017) The future is now: reducing psychological distance 
to increase public engagement with climate change. Risk Analysis: An International Journal 37(2): 331-341.

Kaju A et Maglio SJ (2018) Urgently yours: temporal communication norms and psychological distance. Journal of Consumer Psychology 28(4): 665-672.

Karatas M et Gurhan-Canli Z (2020) When consumers prefer bundles with noncomplementary items to bundles with complementary items: the role of mindset abstraction. Journal of Consumer Psychology 30(1): 24-39.

Keinan A et Kivetz R (2008) Remedying hyperopia: the effects of self-control regret on consumer behavior. Journal of Marketing Research 45(6): 676-689.

Kim BK, Choi J et Wakslak CJ (2019) The image realism effect: the effect of unrealistic product images in advertising. Journal of Advertising 48(3): 251-270.

Kim DH, Sung YH, Lee SY, et al. (2016) Are you on timeline or news feed? The roles of facebook pages and construal level in increasing ad effectiveness. Computers in Human Behavior 57: 312-320.

Kim DH, Sung Y et Drumwright M (2018) 'Where I come from' determines, 'how I construe my future': the fit effect of culture, temporal distance, and construal level. International Journal of Advertising 37(2): 270-288.

Kim H, Rao AR et Lee AY (2009) It's time to vote: the effect of matching message orientation and temporal frame on political persuasion. Journal of Consumer Research 35(6): 877889.

Kim J, Lee S et Rua T (2015) Feeling depleted and powerless: the construal-level mechanism. Personality and Social Psychology Bulletin 41(4): 599-609.

Kim K, Zhang M et Li X (2008) Effects of temporal and social distance on consumer evaluations. Journal of Consumer Research 35(4): 706-713.

Kim K, Lee S et Choi YK (2019) Image proximity in advertising appeals: spatial distance and product types. Journal of Business Research 99: 490-497.

Kim Y-J, Park J et Wyer RS (2009) Effects of temporal distance and memory on consumer judgments. Journal of Consumer Research 36(4): 634-645.

Kivetz R et Keinan A (2006) Repenting hyperopia: an analysis of self-control regrets. Journal of Consumer Research 33(2), 273-282.

Kivetz R et Simonson I (2002) Self-control for the righteous: toward a theory of precommitment to indulgence. Journal of Consumer Research 29(2): 199-217.

Kopetz CE, Kruglanski AW, Arens ZG, et al. (2012) The dynamics of consumer behavior: a goal systemic perspective. Journal of Consumer Psychology 22(2): 208-223.

Kulkarni AA et Yuan H (2015) Effect of ad-irrelevant distance cues on persuasiveness of message framing. Journal of Advertising 44(3): 254-263. 
Kulkarni AA et Yuan H (2017) Construal-level mindsets enhance behavioral persistence in response to incentive valence. Journal of Behavioral Decision Making 30(5): 10411051.

Krpan D et Fasolo B (2019) Revisiting embodied approach and avoidance effects on behavior: the influence of sitting posture on purchases of rewarding foods. Journal of Experimental Social Psychology 85: 1-18.

Kyung EJ, Menon G et Trope Y (2010) Reconstruction of things past: why do some memories feel so close and others so far away? Journal of Experimental Social Psychology 46(1): 217-220.

Kyung EJ, Menon G et Trope Y (2014) Construal level and temporal judgments of the past: the moderating role of knowledge. Psychonomic Bulletin \& Review 21(3): 734-739.

Laran J (2010a) Choosing your future: temporal distance and the balance between self-control and indulgence. Journal of Consumer Research 36(6): 1002-1015.

Laran J (2010b) Goal management in sequential choices: consumer choices for others are more indulgent than personal choices. Journal of Consumer Research 37(2): 304-314.

Lee H, Fujita K, Deng X, et al. (2017) The role of temporal distance on the color of futuredirected imagery: a construal-level perspective. Journal of Consumer Research 43(5): $707-725$.

Lee KK et Zhao M (2014) The effect of price on preference consistency over time. Journal of Consumer Research 41(1): 109-118.

Lenglet $\mathrm{F}$ et Mencarelli R (2020) La proximité en marketing: proposition d'un cadre intégrateur et agenda de recherche. Recherche et Applications en Marketing (French Edition) 35(4) : 103-130.

Leviston Z, Price J et Bishop B (2014) Imagining climate change: the role of implicit associations and affective psychological distancing in climate change responses. European Journal of Social Psychology 44(5): 441-454.

Li K, Lv Y, Dong Y, et al. (2019) Orderliness/disorderliness is mentally associated with construal level and psychological distance. Frontiers in Psychology 10: 1-12.

Liberman N et Förster J (2009) Distancing from experienced self: how global-versus-local perception affects estimation of psychological distance. Journal of Personality and Social Psychology 97(2): 203-216.

Liberman N et Trope Y (1998) The role of feasibility and desirability considerations in near and distant future decisions: A test of temporal construal theory. Journal of personality and social psychology 75(1): 5-18.

Liberman N et Trope Y (2008) The psychology of transcending the here and now. Science 322: 1201-1205.

Liberman N et Trope Y (2014) Traversing psychological distance. Trends in Cognitive Sciences 18(7): 364-369. 
Liberman N, Sagristano MD et Trope Y (2002) The effect of temporal distance on level of mental construal. Journal of Experimental Social Psychology 38(6): 523-534. Liberman N, Trope Y et Wakslak C (2007) Construal level theory and consumer behavior. Journal of Consumer Psychology 17(2): 113-117.

Liberman N, Trope Y et Stephan E (2007) Psychological distance. In: Kruglanski AW et Higgins ET (eds) Social psychology: Handbook of basic principles. The Guilford Press, pp.353-381.

Lin Y-C, Chang CA et Lin Y-F (2012) Self-construal and regulatory focus influences on persuasion: The moderating role of perceived risk. Journal of Business Research 65(8): 1152-1159.

Liu W (2008) Focusing on desirability: The effect of decision interruption and suspension on preferences. Journal of consumer research 35(4): 640-652.

Liviatan I, Trope Y et Liberman N (2008) Interpersonal similarity as a social distance dimension: implications for perception of others' actions. Journal of Experimental Social Psychology 44(5): 1256-1269.

Lorenz MM, Warner RH et VanDeursen MJ (2015) Stated goals and their influence on helping behavior toward ingroups and outgroups. Journal of Applied Social Psychology 45(9): 498-508.

Lu J, Xie X et Xu J (2013) Desirability or feasibility: self-other decision-making differences. Personality and Social Psychology Bulletin 39(2): 144-155.

Lu J, Liu Z et Fang Z (2016) Hedonic products for you, utilitarian products for me. Judgment \& Decision Making 11(4): 332-341.

Lu M et Jen W (2016) Effects of product option framing and temporal distance on consumer choice: the moderating role of process versus outcome mental simulations. Psychology \& Marketing 33(10): 856-863.

MacInnis DJ (2011) A framework for conceptual contributions in marketing. Journal of Marketing 75(4): 136-154.

Magee JC, Milliken FJ et Lurie AR (2010) Power differences in the construal of a crisis: the immediate aftermath of september 11, 2001. Personality and Social Psychology Bulletin 36(3): 354-370.

Magee JC et Smith PK (2013) The social distance theory of power. Personality \& Social Psychology Review (Sage Publications Inc.) 17(2): 158-186.

Maglio SJ (2020) Psychological distance in consumer psychology: consequences and antecedents. Consumer Psychology Review 3(1): 108-125.

Maglio SJ, Trope Y et Liberman N (2013a) Distance from a distance: psychological distance reduces sensitivity to any further psychological distance. Journal of Experimental Psychology-General 142(3): 644-657.

Maglio SJ, Trope Y et Liberman N (2013b) The common currency of psychological distance. 
Current Directions in Psychological Science 22(4): 278-282.

Malkoc SA et Zauberman G (2006) Deferring versus expediting consumption: the effect of outcome concreteness on sensitivity to time horizon. Journal of Marketing Research 43(4): 618-627.

Malkoc SA et Zauberman G (2019) Psychological analysis of consumer intertemporal decisions. Consumer Psychology Review 2(1): 97-113.

Malkoc SA, Zauberman G et Ulu C (2005) Consuming now or later? The interactive effect of timing and attribute alignability. Psychological Science 16(5): 411-417.

Manning C, Mangas H, Amel E, et al. (2018) Psychological distance and response to human versus non-human victims of climate change. In: Leal Filho W, Marans RW, et Callewaert J (eds) Handbook of Sustainability and Social Science Research. World Sustainability Series. Cham: Springer International Publishing, pp. 143-161.

Martin BAS, Gnoth J et Strong C (2009) Temporal construal in advertising: the moderating role of temporal orientation and attribute importance in consumer evaluations. Journal of Advertising 38(3): 5-19.

McCarthy EJ (1964) Basic marketing: a managerial approach. Homewood, IL: Richard D. Irwin.

Melis K, Campo K, Breugelmans E, et al. (2015) The impact of the multi-channel retail mix on online store choice: does online experience matter? Journal of Retailing 91(2): 272288.

Merle A, Herault-Fournier C et Werle COC (2016) The effects of indication of local geographical origin on food perceptions. Recherche et Applications en Marketing (English Edition) 31(1): 26-42.

Metcalfe J et Mischel W (1999) A hot/cool-system analysis of delay of gratification: dynamics of willpower. Psychological review 106(1): 3-19.

Moorman C et Day GS (2016) Organizing for marketing excellence. Journal of Marketing 80(6): 6-35.

Myers TA, Nisbet MC, Maibach EW, et al. (2012) A public health frame arouses hopeful emotions about climate change. Climatic change 113(3-4): 1105-1112.

Nguyen S, Didi Alaoui M et Llosa S (2020) When interchangeability between providers and users makes a difference: The mediating role of social proximity in collaborative services. Journal of Business Research 121: 506-515.

Novak TP et Hoffman DL (2019) Relationship journeys in the internet of things: a new framework for understanding interactions between consumers and smart objects. Journal of the Academy of Marketing Science 47(2): 216-237.

Novak TP, Hoffman DL et Duhachek A (2003) The influence of goal-directed and experiential activities on online flow experiences. Journal of consumer psychology 13(1-2): 3-16. 
Nowlis SM, Mandel N et McCabe DB (2004) The effect of a delay between choice and consumption on consumption enjoyment. Journal of Consumer Research 31(3): 502510 .

O'curry S et Strahilevitz M (2001) Probability and mode of acquisition effects on choices between hedonic and utilitarian options. Marketing Letters 12(1): 37-49.

Paetzold G et Specia L (2016) Inferring psycholinguistic properties of words. In: Proceedings of the 2016 Conference of the North American Chapter of the Association for Computational Linguistics: Human Language Technologies. Stroudsburg, PA: Association for Computational Linguistics: 435-440.

Paramita W, Septianto F, Winahjoe S, et al. (2020) Sharing is (not) caring? The interactive effects of power and psychological distance on tolerance of unethical behavior. Australasian Marketing Journal 28(3): 42-49.

Park S-Y et Morton CR (2015) The role of regulatory focus, social distance, and involvement in anti-high-risk drinking advertising: a construal-level theory perspective. Journal of Advertising 44(4): 338-348.

Park G et Park HS (2016) Corporate social responsibility in korea: how to communicate global issues to local stakeholders. Corporate Social Responsibility \& Environmental Management 23(2): 77-87.

Pennington GL et Roese NJ (2003) Regulatory focus and temporal distance. Journal of Experimental Social Psychology 39(6): 563-576.

Petit O, Velasco C et Spence C (2019) Digital sensory marketing: integrating new technologies into multisensory online experience. Journal of Interactive Marketing 45: 42-61.

Piris Y (2013) Comment réduire la taille d'un assortiment sans affecter la perception de la variété? Une étude des effets isolés de la largeur et de la profondeur d'un assortiment. Recherche et Applications en Marketing (French Edition) 28(3): 45-59.

Pizzi G, Marzocchi GL, Orsingher C, et al. (2015) The temporal construal of customer satisfaction. Journal of Service Research 18(4): 484-497.

Poortinga W, Spence A, Whitmarsh L, et al. (2011) Uncertain climate: an investigation into public scepticism about anthropogenic climate change. Global environmental change 21(3): 1015-1024.

Rickard LN, Yang ZJ et Schuldt JP (2016) Here and now, there and then: how "departure dates" influence climate change engagement. Global Environmental Change 38: 97-107.

Rim S, Min KE, Liu PJ, et al. (2019) The gift of psychological closeness: how feasible versus desirable gifts reduce psychological distance to the giver. Personality and Social Psychology Bulletin 45(3): 360-371.

Roose G, Vermeir I, Geuens M, et al. (2019) A match made in heaven or down under? The effectiveness of matching visual and verbal horizons in advertising. Journal of Consumer Psychology 29(3): 411-427. 
Ryoo Y, Hyun NK et Sung Y (2017) The effect of descriptive norms and construal level on consumers' sustainable behaviors. Journal of Advertising 46(4): 536-549.

Sagristano MD, Trope Y et Liberman N (2002) Time-dependent gambling: odds now, money later. Journal of Experimental Psychology: General 131(3): 364.

Scannell L et Gifford R (2013) Personally relevant climate change: the role of place attachment and local versus global message framing in engagement. Environment and Behavior 45(1): $60-85$.

Schill M et Shaw D (2016) Recycling today, sustainability tomorrow: effects of psychological distance on behavioural practice. European Management Journal 34(4): 349-362.

Schuldt JP, Rickard LN et Yang ZJ (2018) Does reduced psychological distance increase climate engagement? On the limits of localizing climate change. Journal of Environmental Psychology 55: 147-153.

Shapira O, Liberman N, Trope N, et al. (2012) Levels of mental construal. In: Fiske S et McRae N (eds.) Sage handbook of social cognition. Thousand Oaks, CA: Sage Publications, pp. 229-250.

Shiv B et Fedorikhin A (1999) Heart and mind in conflict: the interplay of affect and cognition in consumer decision making. Journal of consumer Research 26(3): 278-292.

Siau K et Long Y (2005) Synthesizing e-government stage models-a meta-synthesis based on meta-ethnography approach. Industrial Management \& Data Systems 105(4): 443-458.

Slepian ML, Masicampo EJ et Ambady N (2015) Cognition from on high and down low: verticality and construal level. Journal of Personality and Social Psychology 108(1): $1-17$.

Smith PK et Trope Y (2006) You focus on the forest when you're in charge of the trees: power priming and abstract information processing. Journal of Personality \& Social Psychology 90(4): 578-596.

Smith PK, Wigboldus DHJ et Dijksterhuis A (2008) Abstract thinking increases one's sense of power. Journal of Experimental Social Psychology 44(2): 378-385.

Spassova G et Lee AY (2013) Looking into the future: a match between self-view and temporal distance. Journal of Consumer Research 40(1): 159-171.

Song D et Kim DH (2020) "I'll donate money today and time tomorrow": the moderating role of attitude toward nonprofit organizations on donation intention. International Journal of Nonprofit and Voluntary Sector Marketing 25(3): 1-9.

Spence A et Pidgeon N (2010) Framing and communicating climate change: the effects of distance and outcome frame manipulations. Global Environmental Change 20(4): 656667.

Spence A, Poortinga W et Pidgeon N (2012) The psychological distance of climate change. Risk Analysis: An International Journal 32(6): 957-972. 
Steinhart Y, Carmon Z et Trope Y (2013) Warnings of adverse side effects can backfire over time. Psychological Science 24(9): 1842-1847.

Stephan E, Liberman N et Trope Y (2010) Politeness and psychological distance: a construal level perspective. Journal of Personality and Social Psychology 98(2): 268-280.

Stillman P, Lee H, Deng X, et al. (2020) Examining consumers' sensory experiences with color: a consumer neuroscience approach. Psychology \& Marketing 37: 995-1007.

Tan TM, Salo J, Juntunen J, et al. (2019) The role of temporal focus and self-congruence on consumer preference and willingness to pay: a new scrutiny in branding strategy. European Journal of Marketing 53(1): 37-62.

Tangari AH et Smith RJ (2012) How the temporal framing of energy savings influences consumer product evaluations and choice. Psychology and Marketing 29(4): 198-208.

Tangari AH, Burton S et Smith RJ (2015) Now that's a bright idea: the influence of consumer elaboration and distance perceptions on sustainable choices. Journal of Retailing 91(3): $410-421$.

Teeny JD, Siev JJ, Briñol P, et al. (2020) A review and conceptual framework for understanding personalized matching effects in persuasion. Journal of Consumer Psychology. Epub ahead of print 15 october 2020. DOI: 10.1002/jcpy.1198.

Thomas M et Tsai CI (2012) Psychological distance and subjective experience: how distancing reduces the feeling of difficulty. Journal of Consumer Research 39(2): 324-340.

Thompson DV, Hamilton RW et Rust RT (2005) Feature fatigue: when product capabilities become too much of a good thing. Journal of marketing research 42(4): 431-442.

Todorov A, Goren A et Trope Y (2007) Probability as a psychological distance: construal and preferences. Journal of Experimental Social Psychology 43(3): 473-482.

Torrez B, Wakslak C et Amit E (2019) Dynamic distance: use of visual and verbal means of communication as social signals. Journal of Experimental Social Psychology 85: 1-8.

Trope Y et Liberman N (2000) Temporal construal and time-dependent changes in preference. Journal of personality and social psychology 79(6): 876-889.

Trope Y et Liberman N (2003) Temporal construal. Psychological Review 110(3): 403-421.

Trope Y et Liberman N (2010) Construal-level theory of psychological distance. Psychological Review 117(2): 440-463.

Trope Y, Liberman N et Wakslak C (2007) Construal levels and psychological distance: effects on representation, prediction, evaluation, and behavior. Journal of consumer psychology 17(2): 83-95.

Vallacher RR et Wegner DM (1989) Levels of personal agency: individual variation in action identification. Journal of Personality and Social psychology 57(4): 660-671.

Van Boven L, White K et Huber M (2009) Immediacy bias in emotion perception: current 
emotions seem more intense than previous emotions. Journal of Experimental Psychology: General 138(3): 368-382.

Van Boven L, Kane J, McGraw AP, et al. (2010) Feeling close: emotional intensity reduces perceived psychological distance. Journal of Personality and Social Psychology 98(6): $872-885$.

Van Kerckhove A, Geuens M et Vermeir I (2015) The floor is nearer than the sky: how looking up or down affects construal level. Journal of Consumer Research 41(6): 1358-1371.

Wakslak C et Trope Y (2009) The effect of construal level on subjective probability estimates. Psychological Science 20(1): 52-58.

Wakslak CJ, Trope Y, Liberman N, et al. (2006) Seeing the forest when entry is unlikely: probability and the mental representation of events. Journal of Experimental Psychology: General 135(4): 641-653.

Wang B, Liu SQ, Kandampully J, et al. (2020) How color affects the effectiveness of tasteversus health-focused advertising messages. Journal of Advertising. Epub ahead of print 3 september 2020. DOI: 10.1080/00913367.2020.1809575.

Wang S, Hurlstone MJ, Leviston Z, et al. (2019) Climate change from a distance: an analysis of construal level and psychological distance from climate change. Frontiers in Psychology 10(230): 1-22.

White K, MacDonnell R et Dahl DW (2011) It's the mind-set that matters: the role of construal level and message framing in influencing consumer efficacy and conservation behaviors. Journal of Marketing Research 48(3): 472-485.

White K, Simpson B et Argo JJ (2014) The motivating role of dissociative out-groups in encouraging positive consumer behaviors. Journal of Marketing Research 51(4): 433447.

White K, Habib R et Hardisty DJ (2019) How to SHIFT consumer behaviors to be more sustainable: a literature review and guiding framework. Journal of Marketing 83(3): 22 49.

Wiesenfeld BM, Reyt J-N, Brockner J, et al. (2017) Construal level theory in organizational research. Annual Review of Organizational Psychology and Organizational Behavior 4: $367-400$.

Williams LE et Bargh JA (2008) Keeping one's distance: the influence of spatial distance cues on affect and evaluation: Research article. Psychological Science 19(3): 302-308.

Williams LE, Stein R et Galguera L (2014) The distinct affective consequences of psychological distance and construal level. Journal of Consumer Research 40(6): 11231138.

$\mathrm{Xu}$ J, Jiang Z et Dhar R (2013) Mental representation and perceived similarity: how abstract mindset aids choice from large assortments. Journal of Marketing Research 50(4): 548559. 
Yadav MS (2010) The decline of conceptual articles and implications for knowledge development. Journal of Marketing 74(1): 1-19.

Yan D (2014) Future events are far away: exploring the distance-on-distance effect. Journal of Personality and Social Psychology 106(4): 514-525.

Yan D et Sengupta J (2011) Effects of construal level on the price-quality relationship. Journal of Consumer Research 38(2): 376-389.

Yan D, Sengupta J et Hong J (2016) Why does psychological distance influence construal level? The role of processing mode. Journal of Consumer Research 43(4): 598-613.

Yoo B et Donthu N (2001) Developing a scale to measure the perceived quality of an Internet shopping site (SITEQUAL). Quarterly journal of electronic commerce 2(1):31-45.

Yoon S-J (2002) The antecedents and consequences of trust in online-purchase decisions. Journal of Interactive Marketing 16(2): 47-63.

Yudkin DA, Pick R, Hur EY, et al. (2019) Psychological distance promotes exploration in search of a global maximum. Personality and Social Psychology Bulletin 45(6): 893906.

Zhang W, He G-B, Zhu Y, et al. (2014) Effects of psychological distance on assessment of severity of water pollution. Social Behavior and Personality: an international journal 42(1): 69-78.

Zhao M, Hoeffler S et Zauberman G (2007) Mental simulation and preference consistency over time: the role of process- versus outcome-focused thoughts. Journal of Marketing Research 44(3): 379-388.

Zhao M, Hoeffler S et Zauberman G (2011) Mental simulation and product evaluation: the affective and cognitive dimensions of process versus outcome simulation. Journal of Marketing Research 48(5): 827-839.

Zhao M et Xie J (2011) Effects of social and temporal distance on consumers' responses to peer recommendations. Journal of Marketing Research 48(3): 486-496.

Ziamou P et Veryzer RW (2005) The influence of temporal distance on consumer preferences for technology-based innovations. Journal of Product Innovation Management 22(4): $336-346$. 


\section{Tableaux}

Tableau 1 : Définitions, sous-types et contextes d'utilisation des quatre dimensions la distance psychologique en marketing

\begin{tabular}{|c|c|c|c|c|}
\hline Dimensions & Définitions & Sous-type & Contextes d'utilisation & Auteurs \\
\hline \multirow[t]{6}{*}{ Temporelle } & \multirow{6}{*}{$\begin{array}{l}\text { Intervalle de } \\
\text { temps entre } \\
\text { le jugement, } \\
\text { l'évaluation } \\
\text { ou le choix } \\
\text { d'un objet et } \\
\text { le moment où } \\
\text { l'objet donné } \\
\text { se réalise }\end{array}$} & $\begin{array}{l}\text { Distance } \\
\text { temporelle } \\
\text { passée }\end{array}$ & $\begin{array}{l}\text { Consommation d'un produit } \\
\text { ou service dans un passé } \\
\text { proche } v s \text {. lointain }\end{array}$ & $\begin{array}{l}\text { Chang et Pham, } 2018 \text {; Grant et Tybout, } \\
2008 \text {; Huang et al., } 2016 \text {; Jin et al., } 2014 \text {; Keinan } \\
\text { et Kivetz, } 2008 \text {; Kivetz et Keinan, } 2006 \text {; Kyung et } \\
\text { al., 2010, } 2014 \text {; Pizzi et al., } 2015 \text {; Tan et al., } 2019\end{array}$ \\
\hline & & \multirow[t]{5}{*}{$\begin{array}{l}\text { Distance } \\
\text { temporelle } \\
\text { future }\end{array}$} & $\begin{array}{l}\text { Consommation d'un produit } \\
\text { ou service dans un futur } \\
\text { proche } v s \text {. lointain }\end{array}$ & $\begin{array}{l}\text { Basoglu et Yoo, 2015 ; Castaño et al., 2008; Chang } \\
\text { et Pham, 2013; Chen et Wyer, 2015; Etkin et } \\
\text { Ratner, 2013; Jeannot et Jolibert, 2013 ; Jin et He, } \\
2013 \text {; Laran 2010a, Lee et al., } 2017 \text {; Lee et Zhao, } \\
\text { 2014; Liberman et Trope, 1998; Trope et } \\
\text { Liberman, } 2003 \text {; Zhao et al., } 2011\end{array}$ \\
\hline & & & $\begin{array}{l}\text { Lancement d'un nouveau } \\
\text { produit dans un futur proche } \\
v s . \text { lointain }\end{array}$ & $\begin{array}{l}\text { Balbo et Jeannot, } 2015 \text {; Bornemann et Homburg, } \\
2011 \text {; Didi Alaoui, } 2020 \text {; Jha et al., } 2019\end{array}$ \\
\hline & & & $\begin{array}{l}\text { Lancement d'une campagne } \\
\text { politique dans un futur } \\
\text { proche } v s . \text { lointain }\end{array}$ & Freitas et al., 2008 ; Kim et al., 2009 \\
\hline & & & $\begin{array}{l}\text { Recevoir un produit en } \\
\text { cadeau dans un futur proche } \\
v s . \text { lointain }\end{array}$ & Lee et al., 2017 ; Williams et al., 2014 \\
\hline & & & $\begin{array}{l}\text { Faire un don pour une action } \\
\text { sociale qui aura lieu dans un } \\
\text { futur proche } v s \text {. lointain }\end{array}$ & $\begin{array}{l}\text { Choi et al., 2012 ; Czeizler et Garbarino, 2017; } \\
\text { Ein-Gar et Levontin, } 2013 \text {; Song et Kim, } 2020 \text {; } \\
\text { Williams et al., } 2014\end{array}$ \\
\hline
\end{tabular}




\begin{tabular}{|c|c|c|c|c|}
\hline & & & $\begin{array}{l}\text { Livraison d'un produit dans } \\
\text { un futur proche } v s \text {. lointain }\end{array}$ & $\begin{array}{l}\text { Cho et al., } 2013 \text {; Kaju et Maglio, } 2018 \text {; Steinhart } \\
\text { et al., } 2013\end{array}$ \\
\hline \multirow[t]{3}{*}{ Spatiale } & \multirow[t]{3}{*}{$\begin{array}{l}\text { Espacement } \\
\text { physique } \\
\text { existant entre } \\
\text { un individu et } \\
\text { un objet } \\
\text { donné }\end{array}$} & $\begin{array}{l}\text { Distance } \\
\text { spatiale } \\
\text { conceptuelle } \\
\text { (imaginer de la } \\
\text { distance } \\
\text { physique entre } \\
\text { soi et un } \\
\text { stimulus) }\end{array}$ & $\begin{array}{l}\text { Choix ou consommation dans } \\
\text { un lieu proche } v s \text {. éloigné du } \\
\text { domicile du consommateur }\end{array}$ & $\begin{array}{l}\text { Darke et al., 2016; Goodman et Malkoc, 2012; } \\
\text { Huang et al., 2016; Jia et Smith, } 2013 \text {; Karatas et } \\
\text { Gürhan-Canli, } 2020 \text {; Merle et al., 2016; Maglio et } \\
\text { al., 2013a ; Ryoo et al., } 2017\end{array}$ \\
\hline & & \multirow{2}{*}{$\begin{array}{l}\text { Distance } \\
\text { spatiale } \\
\text { perceptuelle } \\
\text { (percevoir de } \\
\text { la distance } \\
\text { physique entre } \\
\text { soi et un } \\
\text { stimulus) }\end{array}$} & $\begin{array}{l}\text { Perspective spatiale proche } \\
\text { vs. éloignée des stimuli } \\
\text { présentés }\end{array}$ & $\begin{array}{l}\text { Huang et al., } 2017 \text {; Jia et al., } 2017 \text {; Kim, Lee et } \\
\text { Choi, } 2019 \text {; Williams et Bargh, } 2008 \text {; Yan, } 2014 \text {; } \\
\text { Yudkin et al., } 2019\end{array}$ \\
\hline & & & $\begin{array}{l}\text { Proximité vs. éloignement } \\
\text { physique entre le } \\
\text { consommateur et les stimuli } \\
\text { présentés }\end{array}$ & $\begin{array}{l}\text { Aggarwal et Zhao, } 2015 \text {; Krpan et Fasolo 2019; } \\
\text { Slepian et al., 2015; Thomas et Tsai, 2012 ; Van } \\
\text { Kerckhove et al., } 2015\end{array}$ \\
\hline \multirow[t]{6}{*}{ Sociale } & \multirow{6}{*}{$\begin{array}{l}\text { Distinction } \\
\text { qu'une } \\
\text { personne fait } \\
\text { entre elle- } \\
\text { même et les } \\
\text { autres }\end{array}$} & \multirow[t]{2}{*}{ Le soi } & Achat pour soi vs. un ami & Laran 2010b; Lu et al., 2013, 2016 \\
\hline & & & Recevoir $v s$. offrir un cadeau & $\begin{array}{l}\text { Baskin et al., } 2014 \text {; Choi et al., } 2018 \text {; Rim et al., } \\
2019\end{array}$ \\
\hline & & La similarité & $\begin{array}{l}\text { Choix, don ou évaluation en } \\
\text { lien avec une personne } \\
\text { similaire } v s \text {. dissimilaire }\end{array}$ & $\begin{array}{l}\text { Balbo et al., } 2017 \text {; Ein-Gar et Levontin, } 2013 \text {; } \\
\text { Liviatan et al., } 2008 \text {; Nguyen et al., } 2020\end{array}$ \\
\hline & & Le groupe & $\begin{array}{l}\text { Distinction entre les } \\
\text { personnes de l'endogroupe } \\
\text { vs. l'exogroupe }\end{array}$ & $\begin{array}{l}\text { Liberman, Trope et Stephan, } 2007 \text {; Lorenz et al., } \\
2015 \text {; White et al., } 2014\end{array}$ \\
\hline & & La culture & $\begin{array}{l}\text { Activation du soi } \\
\text { interdépendant } v s \text {. } \\
\text { indépendant }\end{array}$ & $\begin{array}{l}\text { Akpinar et al., } 2018 \text {; Hong et Chang, } 2015 \text {; Lin et } \\
\text { al., } 2012 \text {; Spassova et Lee, } 2013\end{array}$ \\
\hline & & La politesse & Tutoiement vs. vouvoiement & Stephan et al., 2010 \\
\hline
\end{tabular}




\begin{tabular}{|c|c|c|c|c|}
\hline & & Le pouvoir & Pouvoir fort $v s$. faible & $\begin{array}{l}\text { Kim et al., } 2015 \text {; Gawronski et Brannon, } 2020 ; \\
\text { Magee et al., 2010; Magge et Smith, } 2013 ; \\
\text { Paramita et al., } 2020 \text {; Smith et Trope, } 2006\end{array}$ \\
\hline \multirow[t]{2}{*}{ Hypothétique } & \multirow{2}{*}{$\begin{array}{l}\text { Probabilité } \\
\text { d'occurrence } \\
\text { d'un } \\
\text { événement ou } \\
\text { d'une } \\
\text { situation }\end{array}$} & $\begin{array}{l}\text { Degré } \\
\text { d'incertitude }\end{array}$ & $\begin{array}{l}\text { Probabilité forte } v s \text {. faible de } \\
\text { la réalisation d'un événement }\end{array}$ & $\begin{array}{l}\text { Kivetz et Simonson, } 2002 \text {; O’Curry et Strahilevitz, } \\
2001 \text {; Todorov et al., } 2007 \text {; Wakslak et al., 2006; } \\
\text { Williams et al., } 2014\end{array}$ \\
\hline & & $\begin{array}{l}\text { Degré de } \\
\text { réalité }\end{array}$ & Stimuli réels $v s$. fictifs & Bar-Anan et al., 2006 ; Kim, Choi et Wakslak, 2019 \\
\hline
\end{tabular}

Tableau 2 : Les différentes déclinaisons des niveaux de représentation en marketing

\begin{tabular}{|l|l|l|l|}
\hline $\begin{array}{l}\text { Les différentes } \\
\text { opérationnalisations du } \\
\text { changement de niveau de } \\
\text { représentation }\end{array}$ & $\begin{array}{l}\text { Conséquences en situation de } \\
\text { proximité psychologique }\end{array}$ & $\begin{array}{l}\text { Conséquences en situation } \\
\text { d'éloignement psychologique }\end{array}$ & Auteurs \\
\hline $\begin{array}{l}\text { Aspects centraux (haut niveau) } v s . \\
\text { périphériques (bas niveau) d'un } \\
\text { produit }\end{array}$ & $\begin{array}{l}\text { Le consommateur se focalise } \\
\text { davantage sur les attributs } \\
\text { périphériques du produit (ex : } \\
\text { design ; couleur) }\end{array}$ & $\begin{array}{l}\text { Le consommateur se focalise } \\
\text { davantage sur les attributs centraux } \\
\text { du produit (ex : performance, } \\
\text { autonomie) }\end{array}$ & $\begin{array}{l}\text { Trope et Liberman, } \\
2000 ; \text { Kim, Park et } \\
\text { Wyer, 2009 }\end{array}$ \\
\hline $\begin{array}{l}\text { Attributs abstraits (haut niveau) } \\
\text { vs. concrets (bas niveau) d'un } \\
\text { service }\end{array}$ & $\begin{array}{l}\text { Le consommateur se focalise } \\
\text { davantage sur les attributs concrets } \\
\text { d'un service (ex : horaire de } \\
\text { programmation d'une présentation) }\end{array}$ & $\begin{array}{l}\text { Le consommateur se focalise } \\
\text { davantage sur les attributs abstraits } \\
\text { d'un service (ex : clarté de la } \\
\text { présentation) }\end{array}$ & Pizzi et al., 2015 \\
\hline
\end{tabular}




\begin{tabular}{|c|c|c|c|}
\hline $\begin{array}{l}\text { Éléments de désirabilité (haut } \\
\text { niveau) vs. faisabilité (bas niveau) } \\
\text { d'un produit }\end{array}$ & $\begin{array}{l}\text { Le consommateur prête davantage } \\
\text { d'importance aux éléments de } \\
\text { faisabilité du produit (ex : fiabilité, } \\
\text { facilité d'utilisation) }\end{array}$ & $\begin{array}{l}\text { Le consommateur prête davantage } \\
\text { d'importance aux éléments de } \\
\text { désirabilité du produit (ex : qualité, } \\
\text { nombre d'options) }\end{array}$ & $\begin{array}{l}\text { Baskin et al., } 2014 \text {; } \\
\text { Choi et al., } 2018 \text {; } \\
\text { Liberman et Trope, } \\
1998 \text {; Liu, } 2008 \text {; } \\
\text { Lu et al., } 2013 \text {; } \\
\text { Sagristano et al., } \\
2002 \text {; Thompson et } \\
\text { al., } 2005 \text {; Todorov } \\
\text { et al., } 2007 \text {; Van } \\
\text { Kerckhove et al., } \\
2015\end{array}$ \\
\hline $\begin{array}{l}\text { Avantages (haut niveau) vs. } \\
\text { inconvénients (bas niveau) d'un } \\
\text { produit }\end{array}$ & $\begin{array}{l}\text { Le consommateur se concentre } \\
\text { davantage sur les inconvénients } \\
\text { d'un produit (ex : émission de } \mathrm{CO}_{2} \\
\text { d'une voiture) }\end{array}$ & $\begin{array}{l}\text { Le consommateur se concentre } \\
\text { davantage sur les avantages d'un } \\
\text { produit (ex : bon système de } \\
\text { freinage d'une voiture) }\end{array}$ & $\begin{array}{l}\text { Eyal et al. } 2004 ; \\
\text { Herzog et al., } 2007\end{array}$ \\
\hline $\begin{array}{l}\text { Attributs non comparables (haut } \\
\text { niveau) vs. comparables (bas } \\
\text { niveau) d'un produit }\end{array}$ & $\begin{array}{l}\text { Le consommateur prête davantage } \\
\text { d'importance aux attributs } \\
\text { comparables d'un produit (ex : } \\
\text { poids) }\end{array}$ & $\begin{array}{l}\text { Le consommateur prête davantage } \\
\text { d'importance aux attributs non } \\
\text { comparables d'un produit (ex : } \\
\text { arômes) }\end{array}$ & $\begin{array}{l}\text { Cho et al., } 2013 ; \\
\text { Malkoc et al., } 2005\end{array}$ \\
\hline $\begin{array}{l}\text { Similarité (haut niveau) vs. } \\
\text { dissimilarité (bas niveau) entre les } \\
\text { produits formant un ensemble de } \\
\text { considération }\end{array}$ & $\begin{array}{l}\text { Le consommateur perçoit les } \\
\text { différentes alternatives de son } \\
\text { ensemble de considération comme } \\
\text { étant dissimilaires entre-elles (ex : } \\
\text { toutes les montres ne se } \\
\text { ressemblent pas) }\end{array}$ & $\begin{array}{l}\text { Le consommateur perçoit les } \\
\text { différentes alternatives de son } \\
\text { ensemble de considération comme } \\
\text { étant similaires entre-elles (ex : une } \\
\text { montre, ça reste une montre) }\end{array}$ & $\begin{array}{l}\text { Goodman et Malkoc, } \\
2012 \text {; Henderson, } \\
2013\end{array}$ \\
\hline
\end{tabular}




\begin{tabular}{|l|l|l|l|}
\hline $\begin{array}{l}\text { Mode de traitement verbal (haut } \\
\text { niveau) vs. visuel (bas niveau) de } \\
\text { l'information }\end{array}$ & $\begin{array}{l}\text { Le consommateur mobilise plus } \\
\text { facilement des informations } \\
\text { visuelles, sensorielles et } \\
\text { perceptuelles de l'objet (ex : forme, } \\
\text { texture, goût) }\end{array}$ & $\begin{array}{l}\text { Le consommateur mobilise plus } \\
\text { facilement des informations } \\
\text { textuelles et symboliques de l'objet } \\
\text { (ex : ce que cette consommation } \\
\text { signifie pour lui) }\end{array}$ & Yan et al., 2016 \\
\hline $\begin{array}{l}\text { Mode de traitement en noir et } \\
\text { blanc (haut niveau) vs. en } \\
\text { couleurs (bas niveau) de } \\
\text { l'information }\end{array}$ & $\begin{array}{l}\text { Le consommateur se représente } \\
\text { mentalement son environnement } \\
\text { davantage en couleurs }\end{array}$ & $\begin{array}{l}\text { Le consommateur se représente } \\
\text { mentalement son environnement } \\
\text { davantage en noir et blanc }\end{array}$ & $\begin{array}{l}\text { Lee et al., 2017; } \\
\text { Stillman et al., 2020 }\end{array}$ \\
\hline
\end{tabular}

Tableau 3 : Combinaisons entre la distance psychologique et les outils marketing pour maximiser les réponses du consommateur

\begin{tabular}{|c|c|c|c|c|}
\hline $\begin{array}{l}\text { Dimensions de } \\
\text { la distance } \\
\text { psychologique }\end{array}$ & Actions marketing & Variable(s) dépendante(s) & Principaux résultats & Auteurs \\
\hline Temporelle & 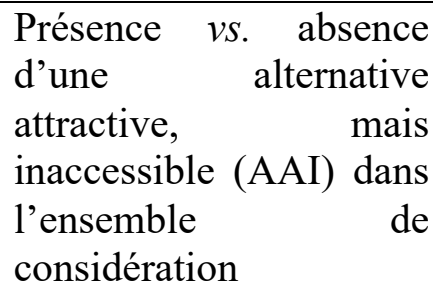 & $\begin{array}{l}\text { 1) Évaluation de l'ensemble } \\
\text { des alternatives }\end{array}$ & $\begin{array}{l}\text { L'ajout d'une AAI améliore (vs. décroit) } \\
\text { l'évaluation de l'ensemble des alternatives } \\
\text { en condition d'éloignement ( } v s \text {. proximité) } \\
\text { temporelle }\end{array}$ & $\begin{array}{l}\text { Borovoi et } \\
\text { al., } 2010\end{array}$ \\
\hline Temporelle & $\begin{array}{l}\text { Cadrage du message } \\
\text { (gains vs. pertes) }\end{array}$ & $\begin{array}{l}\text { 1) Intention d'achat } \\
\text { 2) Consentement à payer }\end{array}$ & $\begin{array}{l}\text { Un message de gains ( } v s . \text { de pertes) associé à } \\
\text { un achat envisagé à long terme ( } v s \text {. court } \\
\text { terme) se présente comme la combinaison la } \\
\text { plus efficace pour promouvoir un produit } \\
\text { innovant }\end{array}$ & $\begin{array}{l}\text { Balbo et } \\
\text { Jeannot, } \\
2015\end{array}$ \\
\hline
\end{tabular}




\begin{tabular}{|c|c|c|c|c|}
\hline Temporelle & $\begin{array}{l}\text { Cadrage du message } \\
\text { (positif } v s . \text { négatif) }\end{array}$ & $\begin{array}{l}\text { 1) Attitude envers une } \\
\text { publicité pour un produit } \\
\text { écologique } \\
\text { 2) Attitude envers la marque } \\
\text { 3) Intention d'achat }\end{array}$ & $\begin{array}{l}\text { Un message de gains (vs. de pertes) tend à } \\
\text { améliorer les réponses du consommateur } \\
\text { envers un produit écologique lorsque le } \\
\text { consommateur pense que les conséquences } \\
\text { de son achat se produiront dans un futur } \\
\text { éloigné ( } v s \text {. proche). }\end{array}$ & $\begin{array}{l}\text { Chang et } \\
\text { al., } 2015\end{array}$ \\
\hline Temporelle & $\begin{array}{l}\text { Terminaison du prix } \\
\text { (ronde } v s \text {. précise) }\end{array}$ & $\begin{array}{l}\text { 1) Attitude envers l'annonce } \\
\text { 2) Attitude envers la marque }\end{array}$ & $\begin{array}{l}\text { Un prix précis (vs. rond) associé à un } \\
\text { événement commercial (ex : lancement d'un } \\
\text { nouveau produit) ayant lieu dans un futur } \\
\text { éloigné ( } v s \text {. proche) améliore les attitudes du } \\
\text { consommateur envers l'annonce et la marque }\end{array}$ & $\begin{array}{l}\text { Didi } \\
\text { Alaoui, } \\
2020\end{array}$ \\
\hline Temporelle & $\begin{array}{l}\text { Nature de l'argument de } \\
\text { persuasion (abstrait vs. } \\
\text { concret) }\end{array}$ & $\begin{array}{l}\text { 1) Attitude envers un } \\
\text { programme de cours } \\
\text { 2) Évaluation d'un produit } \\
\text { 3) Propension à donner à une } \\
\text { association }\end{array}$ & $\begin{array}{l}\text { Un message présentant des arguments } \\
\text { abstraits (vs. concrets) est plus persuasif dans } \\
\text { un contexte d'éloignement (vs. de proximité) } \\
\text { temporel(le) }\end{array}$ & $\begin{array}{l}\text { Fujita et } \\
\text { al., } 2008\end{array}$ \\
\hline $\begin{array}{l}\text { Temporelle } \\
\text { Spatiale }\end{array}$ & $\begin{array}{lcc}\text { Compromis entre } & \text { la } \\
\text { désirabilité } & \text { et } & \text { la } \\
\text { faisabilité (présent } & v s . \\
\text { absent) } & & \end{array}$ & $\begin{array}{l}\text { 1) Évaluation } \quad \text { de } \\
\text { l'assortiment }\end{array}$ & $\begin{array}{l}\text { Lorsque le compromis entre la désirabilité et } \\
\text { la faisabilité est absent (vs. présent), le } \\
\text { consommateur préfère les assortiments de } \\
\text { grande (vs. petite) taille lorsqu'il est en } \\
\text { situation de proximité (vs. éloignement) } \\
\text { temporelle ou spatiale }\end{array}$ & $\begin{array}{l}\text { Goodman } \\
\text { et Malkoc, } \\
2012\end{array}$ \\
\hline Temporelle & $\begin{array}{l}\text { Eléments du produit } \\
\text { (attributs vs. bénéfices) }\end{array}$ & 1) Evaluation du produit & $\begin{array}{l}\text { L'entreprise maximise les réponses du } \\
\text { consommateur lorsqu'elle met en avant les } \\
\text { bénéfices ( } v s \text {. attributs) de son produit et que } \\
\text { l'individu envisage son achat dans un futur } \\
\text { éloigné. }\end{array}$ & $\begin{array}{l}\text { Hernandez, } \\
\text { et al., } 2015\end{array}$ \\
\hline
\end{tabular}




\begin{tabular}{|c|c|c|c|c|}
\hline Spatiale & $\begin{array}{l}\text { Qualité du fit de } \\
\text { l'extension de marque } \\
\text { (bonne } v s . \text { mauvaise) }\end{array}$ & $\begin{array}{l}\text { 1) Évaluation de l'extension } \\
\text { de marque }\end{array}$ & $\begin{array}{l}\text { Le consommateur a une meilleure évaluation } \\
\text { d'une bonne (vs. mauvaise) extension de } \\
\text { marque lorsqu'il expérimente de la proximité } \\
\text { (vs. éloignement) spatial(e) }\end{array}$ & $\begin{array}{l}\text { Huang et } \\
\text { al., } 2017\end{array}$ \\
\hline Temporelle & $\begin{array}{l}\text { Simulation } \quad r \text { mentale } \\
\text { (orientée vers le } \\
\text { processus vs. orientée } \\
\text { vers le résultat) }\end{array}$ & $\begin{array}{l}\text { 1) Incertitude liée aux coûts } \\
\text { d'apprentissage } \\
\text { 2) L'anxiété } \\
\text { 3) L'intention d'utilisation } \\
\text { 4) L'intention d'achat }\end{array}$ & $\begin{array}{l}\text { Dans le cadre de l'adoption d'une nouvelle } \\
\text { technologie, lorsque l'achat est envisagé à } \\
\text { court terme (long terme), la simulation } \\
\text { orientée vers le processus (le résultat) réduit } \\
\text { l'incertitude liée aux coûts d'apprentissage et } \\
\text { l'anxiété. Par ailleurs, elle augmente } \\
\text { l'intention d'utilisation et l'intention d'achat } \\
\text { de la nouvelle technologie }\end{array}$ & $\begin{array}{l}\text { Jeannot et } \\
\text { Jolibert, } \\
2013\end{array}$ \\
\hline Temporelle & $\begin{array}{lr}\text { Descriptions } & \text { des } \\
\text { attributs du service } & \text { (mots } \\
\text { abstraits vs. mots } \\
\text { concrets) }\end{array}$ & $\begin{array}{l}\text { 1) Perception d'utilité du } \\
\text { service } \\
\text { 2) Qualité du service attendue } \\
\text { 3) Intention d'achat }\end{array}$ & $\begin{array}{l}\text { En condition de proximité temporelle, le } \\
\text { consommateur a de meilleures réponses } \\
\text { envers le service lorsque ses attributs sont } \\
\text { décrits avec des mots concrets plutôt } \\
\text { qu'abstraits. En condition d'éloignement } \\
\text { temporel, le consommateur n'exprime pas de } \\
\text { réponses différentes selon la description des } \\
\text { attributs (abstraits } v \text { s. concrets) }\end{array}$ & $\begin{array}{l}\text { Jin et } \mathrm{He} \text {, } \\
2013\end{array}$ \\
\hline Temporelle & $\begin{array}{lr}\begin{array}{l}\text { Lots de produits } \\
\text { (produits }\end{array} \\
\text { complémentaires } & v s . \\
\text { produits } & \text { non } \\
\text { complémentaires) } & \end{array}$ & $\begin{array}{l}\text { 1) Choix entre le lot } \\
\text { complémentaire et non } \\
\text { complémentaire }\end{array}$ & $\begin{array}{l}\text { Le consommateur préfère le lot de produits } \\
\text { complémentaires (vs. non complémentaires) } \\
\text { lorsque la décision implique de la proximité } \\
\text { (vs. éloignement) temporelle. }\end{array}$ & $\begin{array}{l}\text { Karatas et } \\
\text { Gürhan- } \\
\text { Canli, } \\
2020\end{array}$ \\
\hline Spatiale & $\begin{array}{l}\text { Cadrage du message } \\
\text { (rationnel } \\
\text { émotionnel) }\end{array}$ & $\begin{array}{l}\text { 1) Attitude envers la publicité } \\
\text { 2) Attitude envers le produit } \\
\text { 3) Intention d'achat }\end{array}$ & $\begin{array}{l}\text { Le consommateur a de meilleures réponses } \\
\text { lorsque le cadrage du message est rationnel } \\
\text { (vs. émotionnel) et que le produit est présenté } \\
\text { sous un angle proche (éloigné) }\end{array}$ & $\begin{array}{l}\text { Kim, Lee } \\
\text { et Choi, } \\
2019\end{array}$ \\
\hline
\end{tabular}




\begin{tabular}{|c|c|c|c|c|}
\hline Temporelle & $\begin{array}{l}\text { Abstraction du message } \\
\text { (abstrait } v s \text {. concret) }\end{array}$ & $\begin{array}{l}\text { 1) Attitude envers le message } \\
\text { d'un candidat à une élection }\end{array}$ & $\begin{array}{l}\text { Lorsque l'élection aura lieu dans un futur } \\
\text { proche (éloigné), l'individu a une meilleure } \\
\text { attitude envers le message d'un candidat à } \\
\text { l'élection lorsque le message est présenté de } \\
\text { manière concrète ( } v s \text {. abstraite) }\end{array}$ & $\begin{array}{l}\text { Kim et al., } \\
2009\end{array}$ \\
\hline $\begin{array}{l}\text { Spatiale } \\
\text { Sociale }\end{array}$ & $\begin{array}{l}\text { Cadrage du message } \\
\text { (positif vs. négatif) }\end{array}$ & $\begin{array}{l}\text { 1) Attitude envers la publicité } \\
\text { 2) Attitude envers la marque } \\
\text { 3) Intention d'achat }\end{array}$ & $\begin{array}{l}\text { Le consommateur a de meilleures réponses } \\
\text { envers un message cadré positivement ( } v s \text {. } \\
\text { négativement) lorsque ce dernier est associé } \\
\text { à des signaux d'éloignement (vs. de } \\
\text { proximité) spatial(e) ou social(e) }\end{array}$ & $\begin{array}{l}\text { Kulkarni et } \\
\text { Yuan, } \\
2015\end{array}$ \\
\hline Temporelle & $\begin{array}{l}\text { Cadrage de l'option } \\
\text { (additif } v s . \\
\text { soustractif) }\end{array}$ & 1) Difficulté du choix & $\begin{array}{l}\text { Pour une décision qui aura lieu dans un futur } \\
\text { proche (vs. éloigné), le consommateur } \\
\text { expérimente moins de difficulté à opérer son } \\
\text { choix lorsque l'entreprise propose une offre } \\
\text { avec un cadrage soustractif (enlever des } \\
\text { options à l'offre de base) plutôt qu'additif } \\
\text { (rajouter des options à l'offre de base) }\end{array}$ & $\begin{array}{l}\text { Lu et Jen, } \\
2016\end{array}$ \\
\hline Sociale & $\begin{array}{l}\text { Cadrage du message } \\
\text { (gains vs. pertes) }\end{array}$ & $\begin{array}{l}\text { 1) Attitude envers le message } \\
\text { 2) Attitude envers le } \\
\text { comportement plébiscité } \\
\text { 3) Intention comportementale }\end{array}$ & $\begin{array}{l}\text { Le consommateur sera davantage persuadé } \\
\text { par une publicité préventive lorsque } \\
\text { l'émetteur du message est socialement } \\
\text { éloigné du consommateur et que l'annonce } \\
\text { met l'accent sur les gains plutôt que sur les } \\
\text { pertes que le consommateur obtiendra s'il } \\
\text { adopte le comportement plébiscité par le } \\
\text { message. }\end{array}$ & $\begin{array}{l}\text { Park et } \\
\text { Morton, } \\
2015\end{array}$ \\
\hline Temporelle & $\begin{array}{l}\text { Disposition de la photo } \\
\text { du message (horizon bas } \\
v s \text {. horizon haut) }\end{array}$ & $\begin{array}{l}\text { 1) Attractivité du message } \\
\text { 2) Intention d'achat }\end{array}$ & $\begin{array}{l}\text { L'adéquation entre un message présenté dans } \\
\text { une photo avec horizon haut ( } v s \text {. bas) et une } \\
\text { perspective temporelle proche ( } v s \text {. éloignée) } \\
\text { renforce la fluidité de traitement du message } \\
\text { qui, par la suite, influence positivement }\end{array}$ & $\begin{array}{l}\text { Roose et } \\
\text { al., } 2019\end{array}$ \\
\hline
\end{tabular}




\begin{tabular}{|c|c|c|c|c|}
\hline & & & $\begin{array}{l}\text { l'attractivité du message et l'intention } \\
\text { d'achat. }\end{array}$ & \\
\hline Spatiale & $\begin{array}{l}\text { Cadrage du message } \\
\text { (abstrait vs. concret) }\end{array}$ & $\begin{array}{l}\text { 1) Intention de participer à } \\
\text { une campagne de } \\
\text { développement durable }\end{array}$ & $\begin{array}{l}\text { Les normes locales comparativement à des } \\
\text { normes nationales sont plus efficaces lorsque } \\
\text { le message concernant une campagne de } \\
\text { développement durable est présenté de } \\
\text { manière concrète. Lorsque le message est } \\
\text { présenté de manière abstraite, les deux types } \\
\text { de normes ont des résultats équivalents. }\end{array}$ & $\begin{array}{l}\text { Ryoo et al., } \\
2017\end{array}$ \\
\hline Temporelle & $\begin{array}{l}\text { Cadrage du message } \\
\text { (gains } v s . \text { pertes) }\end{array}$ & 1) Intention de recycler & $\begin{array}{l}\text { Un message de gains ( } v s \text {. pertes) associé à } \\
\text { une action effectuée dans le futur ( } v s \text {. } \\
\text { présent) maximise l'intention de recycler du } \\
\text { consommateur. }\end{array}$ & $\begin{array}{l}\text { White et } \\
\text { al., } 2011\end{array}$ \\
\hline
\end{tabular}




\section{Annexes}

Annexe 1 : Stratégies et actions managériales pour gérer la distance psychologique du consommateur

\begin{tabular}{|c|c|c|c|c|c|c|}
\hline & \multicolumn{3}{|c|}{ Stratégie 1 : Réduction de la distance psychologique } & \multicolumn{3}{|c|}{ Stratégie 2 : Augmentation de la distance psychologique } \\
\hline $\begin{array}{l}\text { Dimensions de } \\
\text { la distance } \\
\text { psychologique }\end{array}$ & Objectif & Actions & Exemples d'entreprises & Objectif & Actions & Exemples d'entreprises \\
\hline Temporelle & $\begin{array}{l}\text { Réduire } \\
\text { l'attente du } \\
\text { consommateur }\end{array}$ & $\begin{array}{l}\text { 1) Réduire le délai de } \\
\text { livraison, de mise en } \\
\text { service } \\
\text { 2) Réduire les files } \\
\text { d'attente, proposer } \\
\text { des billets coupe-file } \\
\text { 3) Réduire le délai de } \\
\text { lancement d'un } \\
\text { nouveau produit sur } \\
\text { le marché } \\
\text { 4) Mettre en place le } \\
\text { système : acheter } \\
\text { maintenant, payer } \\
\text { plus tard } \\
\text { 5) Proposer une offre } \\
\text { groupée version } \\
\text { numérique/physique }\end{array}$ & $\begin{array}{l}\text { - Le service Darty Plus } \\
\text { propose une livraison } \\
\text { express en } 2 \mathrm{~h} \text { chrono avec } \\
\text { possibilité de rendez-vous. } \\
\text { - Orange s'est équipé d'un } \\
\text { logiciel de gestion de } \\
\text { rendez-vous qui réduit } \\
\text { l'attente du client. } \\
\text { - Ryanair propose le } \\
\text { service Flexi Plus avec } \\
\text { l'embarquement prioritaire } \\
\text { et le Fast Track pour utiliser } \\
\text { une ligne de contrôle } \\
\text { sécurité dédiée. } \\
\text { - L'Oréal a créé le club des } \\
\text { L'Oréalistas permettant de } \\
\text { recevoir en avant-première } \\
\text { les nouveautés de la } \\
\text { marque. an objet } \\
\text { - Paypal propose le service } \\
\text { paiement après livraison qui } \\
\text { permet au consommateur } \\
\text { d'acquérir un an a }\end{array}$ & $\begin{array}{l}\text { Accroître } \\
\text { l'attente du } \\
\text { consommateur }\end{array}$ & $\begin{array}{l}\text { 1) Augmenter le } \\
\text { temps d'accès à } \\
\text { l'offre } \\
\text { 2) Augmenter } \\
\text { l'attente dans les } \\
\text { files } \\
\text { 3) Mettre en place le } \\
\text { système : Payer } \\
\text { maintenant, } \\
\text { consommer plus } \\
\text { tard } \\
\text { 4) Retarder le } \\
\text { lancement du } \\
\text { nouveau produit }\end{array}$ & $\begin{array}{l}\text { - Obtenir une table au } \\
\text { restaurant Noma à } \\
\text { Copenhague demande près } \\
\text { de } 6 \text { mois d'attente. } \\
\text { - Il faut attendre plus de } 20 \\
\text { ans pour devenir membre du } \\
\text { Bohemian Club, l'un des } \\
\text { clubs politiques américains } \\
\text { les plus fermés du monde. } \\
\text { - Crowdfarming offre au } \\
\text { consommateur la possibilité } \\
\text { d'adopter un arbre et de } \\
\text { recevoir plus tard les fruits } \\
\text { récoltés de cet arbre. } \\
\text { - Il faut patienter près d'une } \\
\text { demi-heure devant un } \\
\text { magasin Abercrombie \& } \\
\text { Fitch pour pouvoir y rentrer. } \\
\text { - Smartbox permet au } \\
\text { consommateur d'acheter une } \\
\text { prestation à un instant t et de } \\
\text { la consommer en t+1. }\end{array}$ \\
\hline
\end{tabular}




\begin{tabular}{|c|c|c|c|c|c|c|}
\hline & & & $\begin{array}{l}\text { immédiatement et de le } \\
\text { payer plus tard. } \\
\text { - Amazon propose une } \\
\text { version groupée livres } \\
\text { numérique et papier pour } \\
\text { réduire le temps d'attente du } \\
\text { consommateur. }\end{array}$ & & & $\begin{array}{l}\text { - Samsung a retardé le } \\
\text { lancement de son nouveau } \\
\text { téléphone Galaxy Z Fold } 2 .\end{array}$ \\
\hline Spatiale & $\begin{array}{l}\text { Réduire } \\
\text { l'espace } \\
\text { physique entre } \\
\text { le } \\
\text { consommateur } \\
\text { et l'offre }\end{array}$ & $\begin{array}{l}\text { 1) Proposer des points } \\
\text { de contact de } \\
\text { proximité } \\
\text { 2) Placer le produit } \\
\text { dans le linéaire en } \\
\text { niveau } 3 \text { ou } 4 \\
\text { 3) Mettre le produit } \\
\text { sur un présentoir en } \\
\text { accès libre } \\
\text { 4) Proposer un essai } \\
\text { en réel ou une } \\
\text { dégustation du } \\
\text { produit }\end{array}$ & $\begin{array}{l}\text { - Carrefour a développé } \\
\text { l'enseigne Carrefour City } \\
\text { pour proposer son offre au } \\
\text { cœur des villes. } \\
\text { - Carglass propose ses } \\
\text { prestations directement } \\
\text { chez le consommateur ou } \\
\text { sur son lieu de travail. } \\
\text { - Les produits de marques } \\
\text { de distributeurs (MDD) } \\
\text { sont très souvent placé en } \\
\text { niveau } 3 \text { et } 4 \text { dans le } \\
\text { linéaire. } \\
\text { - La Fnac place ses } \\
\text { produits électroniques sur } \\
\text { présentoir pour que le } \\
\text { consommateur puisse les } \\
\text { toucher et les essayer. } \\
\text { - La Cure Gourmande } \\
\text { propose au consommateur } \\
\text { de nombreuses dégustations } \\
\text { de ses produits. }\end{array}$ & $\begin{array}{l}\text { Accroître } \\
\text { l'espace } \\
\text { physique entre } \\
\text { le consommateur } \\
\text { et l'offre }\end{array}$ & $\begin{array}{l}\text { 1) Proposer des } \\
\text { points de contact } \\
\text { virtuel } \\
\text { 2) Placé le produit } \\
\text { dans le linéaire en } \\
\text { niveau } 5 \text { ou } 6 \\
\text { 3) Placer le produit } \\
\text { dans une vitrine } \\
\text { individuelle } \\
\text { d'exposition } \\
\text { 4) Proposer un essai } \\
\text { virtuel du produit }\end{array}$ & $\begin{array}{l}\text { - L'offre Red by SFR } \\
\text { propose un service client } \\
\text { uniquement virtuel. } \\
\text { - Les produits de marques } \\
\text { nationales sont très souvent } \\
\text { placés en niveau } 5 \text { ou } 6 \text { dans } \\
\text { le linéaire. } \\
\text { - Le magasin Louis Vitton } \\
\text { des Champs Elysées } \\
\text { présente certains de ces } \\
\text { produits en vitrine. } \\
\text { - Mercedes propose un essai } \\
\text { virtuel de certains de ses } \\
\text { modèles au moyen de la } \\
\text { réalité augmentée. }\end{array}$ \\
\hline
\end{tabular}




\begin{tabular}{|c|c|c|c|c|c|c|}
\hline Sociale & $\begin{array}{l}\text { Réduire la } \\
\text { dissimilarité } \\
\text { entre la } \\
\text { marque et le } \\
\text { consommateur }\end{array}$ & $\begin{array}{l}\text { 1) Inciter le } \\
\text { consommateur à se } \\
\text { recentrer sur soi } \\
\text { 2) Utiliser dans la } \\
\text { publicité des } \\
\text { personnes similaires } \\
\text { au consommateur } \\
\text { 3) Mettre en scène } \\
\text { l'endogroupe (ex : } \\
\text { famille) } \\
\text { 4) Favoriser la } \\
\text { proximité entre des } \\
\text { cultures différentes } \\
\text { 5) Utilisation du } \\
\text { tutoiement } \\
\text { 6) Redonner du } \\
\text { pouvoir au } \\
\text { consommateur }\end{array}$ & $\begin{array}{l}\text { - Mars dans l'une de ses } \\
\text { publicités sur les barres } \\
\text { glacées met l'accent sur } \\
\text { l'importance de penser } \\
\text { d'abord à soi (il n'y a pas } \\
\text { de mal à ne pas partager). } \\
\text { - Aldi dans sa publicité « la } \\
\text { vraie surprise de Noël » } \\
\text { met en scène des vrais } \\
\text { consommateurs qui } \\
\text { essayent certains produits } \\
\text { de la marque. } \\
\text { - Nutella a développé } \\
\text { plusieurs spots publicitaires } \\
\text { autour de la famille dont « } \\
\text { les recettes en famille ». } \\
\text { - Daniel Barenboïm, un } \\
\text { pianiste et un chef } \\
\text { d'orchestre } \\
\text { internationalement connu, a } \\
\text { créé un orchestre mixte } \\
\text { pour promouvoir un } \\
\text { dialogue entre Juifs et } \\
\text { Musulmans. Il encourage à } \\
\text { travers la musique à créer } \\
\text { une proximité entre } \\
\text { cultures différentes. } \\
\text { - Betclic a développé tout } \\
\text { une série d'affiches } \\
\text { publicitaires où la marque } \\
\text { tutoie le consommateur (ex } \\
\text { : quand tu as } 100 \text { euros } \\
\text { offerts). }\end{array}$ & $\begin{array}{l}\text { Accroître la } \\
\text { dissimilarité } \\
\text { entre } \\
\text { la marque et le } \\
\text { consommateur }\end{array}$ & $\begin{array}{l}\text { 1) Inciter le } \\
\text { consommateur à se } \\
\text { recentrer sur l'autre } \\
\text { 2) Utiliser dans la } \\
\text { publicité des } \\
\text { personnes } \\
\text { dissimilaires au } \\
\text { consommateur } \\
\text { 3) Utiliser une } \\
\text { langue étrangère } \\
\text { 4) Favoriser la } \\
\text { sélectivité du } \\
\text { consommateur } \\
\text { 5) Utilisation du } \\
\text { vouvoiement }\end{array}$ & $\begin{array}{l}\text { - Kinder Bueno a } \\
\text { développé de nombreuses } \\
\text { publicités autour du partage } \\
\text { avec l'autre. } \\
\text { - Sony a utilisé comme } \\
\text { égérie Hatsune Miku la diva } \\
\text { pop virtuelle japonaise. } \\
\text { - Bouygues Telecom a } \\
\text { pendant longtemps utilisé le } \\
\text { slogan «We love } \\
\text { technology». } \\
\text { - Black up marque de } \\
\text { cosmétiques spécialement } \\
\text { dédiés aux peaux mates à } \\
\text { foncées, éloigne clairement } \\
\text { les consommateurs qui ne } \\
\text { font pas partie de sa cible. } \\
\text { - De très nombreuses } \\
\text { entreprises utilisent le } \\
\text { « vous » pour s'adresser au } \\
\text { consommateur. }\end{array}$ \\
\hline
\end{tabular}




\begin{tabular}{|c|c|c|c|c|c|c|}
\hline & & & $\begin{array}{l}\text { - La marque C'est qui le } \\
\text { patron ?! redonne du } \\
\text { pouvoir au consommateur } \\
\text { pour qu'il reprenne le } \\
\text { contrôle sur la } \\
\text { consommation. }\end{array}$ & & & \\
\hline Hypothétique & $\begin{array}{l}\text { Réduire } \\
\text { l'incertitude } \\
\text { du } \\
\text { consommateur }\end{array}$ & $\begin{array}{l}\text { 1) Rassurer le } \\
\text { consommateur } \\
\text { 2) Mise en place du } \\
\text { système de pré- } \\
\text { commande } \\
\text { 3) Augmenter la } \\
\text { probabilité } \\
\text { d'occurrence de } \\
\text { l'événement }\end{array}$ & $\begin{array}{l}\text { - La Redoute a repensé son } \\
\text { site pour réduire } \\
\text { l'incertitude du e-client en } \\
\text { développant des visuels, } \\
\text { des liens et du texte } \\
\text { destinés à le rassurer. } \\
\text { - Avec son système de pré- } \\
\text { commandes, Micromania } \\
\text { réduit l'incertitude du } \\
\text { consommateur quant à } \\
\text { l'obtention du produit le } \\
\text { jour de sa sortie. } \\
\text { - La Française des jeux a } \\
\text { lancé le jeu de hasard « Olé } \\
\text { Sombrero » offrant une } \\
\text { probabilité de gagner (= } 35 \\
\%) \text { bien supérieure à } \\
\text { d'autres jeux classiques } \\
\text { comme « Vegas » (= } 25 \\
\%) \text {. }\end{array}$ & $\begin{array}{l}\text { Accroître } \\
\text { l'incertitude du } \\
\text { consommateur }\end{array}$ & $\begin{array}{l}\text { 1) Mettre en place } \\
\text { des ventes flashs } \\
\text { 3) Mettre en place } \\
\text { des ventes aux } \\
\text { enchères } \\
\text { 4) Réduire la } \\
\text { probabilité } \\
\text { d'occurrence d'un } \\
\text { événement }\end{array}$ & $\begin{array}{l}\text { - À l'occasion du Black } \\
\text { Friday, différents slogans } \\
\text { comme « dépêchez-vous ! Il } \\
\text { n'y en aura pas pour tout le } \\
\text { monde » ont magnifié le } \\
\text { caractère hypothétique de } \\
\text { l'achat. } \\
\text { - Le site Interenchères a } \\
\text { démocratisé la vente aux } \\
\text { enchères en ligne qui } \\
\text { s'apparente à une pratique } \\
\text { d'achat hautement } \\
\text { hypothétique. } \\
\text { - Sony annonce que tout le } \\
\text { monde ne pourra pas acheter } \\
\text { ni même trouver une PS5 le } \\
\text { jour de son lancement. }\end{array}$ \\
\hline
\end{tabular}


Annexe 2 : Outils pour identifier les modalités de bas ou haut niveau des variables du marketing-mix

\begin{tabular}{|c|c|c|}
\hline Outils & Principes & Identifier la modalité de bas et haut niveau \\
\hline $\begin{array}{l}\text { Behavioral } \\
\text { Identification Form } \\
\text { (BIF, Vallacher et } \\
\text { Wegner, 1989) }\end{array}$ & $\begin{array}{l}\text { 1) Exposer la moitié des répondants à une modalité de la variable } \\
\text { du mix étudié. Exposer l'autre moitié des répondants à l'autre } \\
\text { modalité (ex: prix ronds vs précis ; attributs utilitaires vs } \\
\text { hédoniques ; cadrage positif vs négatif ; assortiment petit vs large). } \\
\text { 2) Poser au répondant les } 25 \text { questions du BIF qui l'invitent à } \\
\text { choisir entre deux phrases (a ou b) pour décrire une action (ex : } \\
\text { prendre soin des plantes d'intérieur : a) rendre la pièce jolie ou b) } \\
\text { arroser les plantes). }\end{array}$ & $\begin{array}{l}\text { 1) Coder les descriptions du } \boldsymbol{B I F} \text { selon leur niveau de représentation : } \\
\text { réponse a) (rendre la pièce jolie) }=\text { haut niveau }=0 \text {; réponse b) (arroser } \\
\text { les plantes) = bas niveau }=1 \text {. Puis faire la somme pour chaque } \\
\text { répondant. } \\
\text { 2) Faire un test de différence de moyenne entre les deux conditions de } \\
\text { la variable du mix étudiée (modalité } 1 \text { vs modalité } 2 \text { ). Si le test } \\
\text { statistique est significatif alors la modalité de bas (haut) niveau sera la } \\
\text { condition où le score moyen sera le plus (moins) élevé. }\end{array}$ \\
\hline $\begin{array}{l}\text { Tâche de } \\
\text { catégorisation } \\
\text { (Liberman et al., } \\
\text { 2002) }\end{array}$ & $\begin{array}{l}\text { 1) Exposer la moitié des répondants à une modalité de la variable } \\
\text { du mix étudié. Exposer l'autre moitié des répondants à l'autre } \\
\text { modalité. } \\
\text { 2) Présenter aux participants } 38 \text { objets de la vie courante (ex : } \\
\text { vase, tapis, réfrigérateur, télévision, etc.). } \\
\text { 3) Demander au répondant de catégoriser les } 38 \text { objets. Le } \\
\text { répondant peut créer autant de catégories qu'il souhaite sans } \\
\text { utiliser deux fois le même objet. }\end{array}$ & $\begin{array}{l}\text { 1) Faire la moyenne du nombre de catégories formées par les } \\
\text { répondants pour chacune des modalités du mix. } \\
\text { 2) Faire un test de différence de moyenne entre les deux conditions de } \\
\text { la variable du mix étudiée (modalité } 1 \text { vs modalité } 2 \text { ). Si le test } \\
\text { statistique est significatif, alors la modalité de bas (haut) niveau sera } \\
\text { la condition où le nombre moyen de catégorie sera le plus (moins) } \\
\text { élevé. Les individus sur un bas (haut) niveau tendent à être davantage } \\
\text { exclusifs (inclusifs) dans leur classement et à former plus (moins) de } \\
\text { catégories. }\end{array}$ \\
\hline $\begin{array}{l}\text { Niveau de } \\
\text { concrétisation du } \\
\text { langage (Humphreys } \\
\text { et al., 2020) }\end{array}$ & $\begin{array}{l}\text { 1) Exposer la moitié des répondants à une modalité de la variable } \\
\text { du mix étudié. Exposer l'autre moitié des répondants à l'autre } \\
\text { modalité. } \\
\text { 2) Demander au participant d'écrire un texte relatif à la variable du } \\
\text { mix étudiée (ex : qu'est-ce que le prix en général représente pour } \\
\text { vous?). }\end{array}$ & $\begin{array}{l}\text { 1) Calculer le score de concrétisation du langage de chaque répondant } \\
\text { en analysant le texte de ce dernier via l'outil « construal scoring tool } \\
\text { " (www.construalscore.com). } \\
\text { 2) Faire la moyenne des scores de concrétisation par modalité de la } \\
\text { variable du mix étudiée. } \\
\text { 3) Faire un test de différence de moyenne entre les deux conditions de } \\
\text { la variable du mix (modalité } 1 \text { vs modalité } 2 \text { ). Si le test statistique est } \\
\text { significatif alors la modalité de bas (haut) niveau sera la condition où } \\
\text { le score } M R C \text { de Paetzold et Specia (2016) sera le plus (moins) élevé. }\end{array}$ \\
\hline
\end{tabular}

